TRANSACTIONS OF THE

AMERICAN MATHEMATICAL SOCIETY

Volume 348, Number 8, August 1996

\title{
HALF DE RHAM COMPLEXES AND LINE FIELDS ON ODD-DIMENSIONAL MANIFOLDS
}

\author{
HOUHONG FAN
}

\begin{abstract}
In this paper we introduce a new elliptic complex on an odddimensional manifold with a self-dual line field. The notion of a self-dual line field is a generalization of the notion of a conformal line field. Ellipticity, Fredholm properties and Hodge decompositions of these new complexes are proved both in the case of a closed manifold and in the case of a manifold with boundary. The cohomology groups of these elliptic complexes are computed in some cases. In addition, in this paper, we generalize the notion of an antiself-dual connection on a smooth 4-manifold to a 3-manifold with a line field and a smooth 5-manifold with a line field. The above new elliptic complexes can be twisted by anti-self-dual connections in dimensions 3 and 5 , but only by flat connections in dimensions above 5 . This reveals a special feature of dimensions 3 and 5 .
\end{abstract}

\section{INTRODUCTION}

In this paper, we study odd-dimensional manifolds with smooth tangent line fields. The main point of this paper is that there is a new elliptic complex on an odd-dimensional manifold with a self-dual line field. The notion of a self-dual line field is a generalization of the notion of a conformal line field. In paper [7], we have developed the corresponding index theory on a compact smooth odd-dimensional manifold with boundary and with a general line field which is transverse to the boundary. But when the line field is self-dual, this index theory can be refined as the cohomology theory of the new elliptic complex, which we shall study in this paper. Moreover many of the topological inequalities for a general line field found in [7] become equalities when the line field is self-dual. The new elliptic complexes are called half De Rham complexes; their cohomology groups are called self-duality cohomology groups.

In the orientable case, a line field is generated by a nowhere zero vector field. We are interested in studying the interactions of gauge fields, flows, and the topology of the underlying manifold. For further progress in this direction, see [7], [8], [9].

In most of the constructions in this paper, we need a tangent codimension-one plane field $L$ which is transverse to the line field. But we should like to emphasize that in all sections of this paper except in Section 8, we do not assume that $L$ is integrable: it can be any smooth tangent codimension-one plane field which is transverse to the line field. When the manifold is equipped with a Riemannian metric, we just simply take the orthogonal complement of the line field as $L$, and the

Received by the editors March 23, 1995 and, in revised form, November 6, 1995.

1991 Mathematics Subject Classification. Primary 57R25, 57M99; Secondary 57R80, 58F25, $58 \mathrm{~A} 12$. 
main body of our results are valid in this setting. Section 8 concerns some technical computations of the middle-dimensional self-duality cohomology groups in special cases. These results in special cases are used in our paper [7] to determine the dimensions of the self-duality cohomology groups in the most general case, which of course includes the case that $L$ is non-integrable.

Another point of this paper is that we generalize the notion of an anti-self-dual connection on a smooth 4-manifold to a smooth 5-manifold with a line field and a 3-manifold with a line field. The half De Rham complexes are well-defined for twistings of anti-self-dual connections in dimensions 5 and 3 , but only for twistings of flat connections in dimensions above 5 . This simple fact maybe is a glimpse of rich structures and interactions in dimensions 5 and 3.

This paper is organized as follows. In the first two sections, we present the constructions of real and complex half De Rham complexes on an odd-dimensional manifold with a line field. In Section 3, we give the proofs that the half De Rham complexes are well-defined and elliptic. In Section 4, we study the self-duality cohomology groups on a closed oriented odd-dimensional manifold. Applying the Atiyah-Singer index theorem, we obtain universal relations for the dimensions of self-duality cohomology groups. In Section 5, we study half De Rham complexes on a compact oriented manifold with boundary in the case the line field is transverse to the boundary. We put boundary conditions on the complexes, which are essentially combinations of Neumann and Dirichlet boundary conditions. These boundary conditions are locally elliptic, a fact which is proved in Section 9. Therefore the half De Rham complexes with these boundary conditions have all the standard elliptic and Fredholm properties. In Section 6, we discuss the notion of a self-dual line field. The half De Rham complexes are well-defined on any odd-dimensional manifold with a self-dual line field. In dimensions 3 and 5 , this notion is equivalent to the notion of a conformal line field. But in odd-dimensions above 5 , it seems rather weaker than the notion of a conformal line field. In Section 7 , we compute the $(m-1)$-th self-duality cohomology groups in a general case, and also get an inequality about the dimensions of the $(m+1)$-th self-duality cohomology groups in a general case. In Section 8, we compute the $(m+1)$-th and the $m$-th self-duality cohomology groups in some special cases. As a by-product of the arguments, we obtain the following theorem, valid in all dimensions: If a connected, compact oriented smooth cobordism admits a codimension-one geodesible foliation, which is defined by a nowhere zero closed 1-form, then the cobordism must be a rationalhomology-h-cobordism. (See Theorem 8.9.) Section 9 contains all the postponed computational proofs.

Acknowledgment. This paper is based on ideas in the first part of my University of Chicago Ph.D. thesis. I am most grateful to Professor Melvin G. Rothenberg for his encouragement and guidance. I am also greatly indebted to Professor Clifford H. Taubes for many helpful communications and discussions. I wish to express my thanks to Professors Robert E. Gompf, Ronnie Lee and Tom S. Mrowka for kind conversations. Finally I express my particular gratitude to Professor William S. Massey for his linguistic revising of the whole manuscript.

\section{Real half De Rham complexes}

Let $M$ be a smooth $n$-manifold, and let $H$ be a smooth tangent line field over $M$. Then $H$ induces a dual cotangent codimension-one plane field $L^{*}$ over $M$ by 
$L^{*} \stackrel{\text { def }}{=} H^{\perp}$, where the $\perp$ is with respect to the pairing of a vector and covector at the same point. An element of $H$ is called a flow vector, and an element of $\wedge^{j} L^{*}$ is called a level $j$-covector. A section of $\wedge^{j} L^{*}$ is called a real level $j$-form. In this section we let $\Omega_{l}^{j}(M)$ denote the space of all smooth real level $j$-forms on $M$, and let $\Omega^{j}(M)$ denote the space of all smooth real $j$-forms on $M$. All differential forms, connections, sections of bundles, line fields and plane fields are assumed to be smooth and all metrics are assumed to smooth positive-definite in this paper.

For any form $\omega$ and any vector $X \in H, i_{X} \omega$ is always a level form, where $i_{X}$ denotes the interior contraction. This is because $\left\langle i_{X} \omega, Y\right\rangle=\langle\omega, X \wedge Y\rangle=0$ for $X, Y \in H$ since $\operatorname{dim} H=1$. For any vector $X \in H$, we define the operator

$$
\nabla_{X} \stackrel{\text { def }}{=} i_{X} d: \Omega^{j}(M) \rightarrow \Omega_{l}^{j-1}(M),
$$

which sends forms to level forms of the same degree. Recall that the Lie derivative $L_{X}=i_{X} d+d i_{X}$. Therefore, we have $\nabla_{X} \alpha=L_{X} \alpha$ for level forms $\alpha$ and $X \in H$. In particular, $L_{f X} \alpha=f L_{X} \alpha$ for any level form $\alpha$.

Let $E$ be a real or complex vector bundle over $M$. In this section, we let $\Omega_{l}^{j}(E)$ denote the space of sections of $\wedge^{j} L^{*} \otimes_{\mathbb{R}} E$, and let $\Omega^{j}(E)$ denote the space of $E$-valued real $j$-forms on $M$. Let $A$ be a connection on $E$. Define

$$
\nabla_{A_{X}} \stackrel{\text { def }}{=} i_{X} d_{A}: \Omega^{j}(E) \rightarrow \Omega_{l}^{j-1}(E) .
$$

The curvature $F_{A}$ of $A$ is a $H o m_{\mathbb{R}}(E, E)$-valued or $\operatorname{Hom}_{\mathbb{C}}(E, E)$-valued real 2-form. For a vector $X \in H$, we define

$$
F_{A_{X}} \stackrel{\text { def }}{=} i_{X} F_{A} .
$$

Note that $F_{A_{X}}$ is a $H o m_{\mathbb{R}}(E, E)$-valued or $H_{0} m_{\mathbb{C}}(E, E)$-valued level real 1-form.

Suppose $L^{*}$ is given an orientation $o_{l}$ and a fiberwise metric $g_{l}$. Then we have the level-star operator $*_{l}: \wedge^{j} L^{*} \rightarrow \wedge^{n-1-p} L^{*}$, which is defined by the identity $\alpha \wedge *_{l} \beta=\langle\alpha, \beta\rangle_{g_{l}} \operatorname{vol}\left(g_{l}\right)$ for all $\alpha, \beta \in \wedge^{j} L^{*}$. An orientation and a metric of $L^{*}$ are called a level-orientation and a level-metric respectively.

Now let us assume $\operatorname{dim} M=2 m+1$, where $m$ is a positive integer. To measure non-commutativity of $\nabla_{X}$ and $*_{l}$, we define the level-star-torsion operator $T_{X}$ by

$$
T_{X} \stackrel{\text { def }}{=} \nabla_{X} *_{l}-*_{l} \nabla_{X}=L_{X} *_{l}-*_{l} L_{X}: \Omega_{l}^{j}(M) \rightarrow \Omega_{l}^{j}(M) .
$$

It is easy to see that $T_{X}$ is a zero-th order linear operator. Thus it is well-defined on $\wedge^{j} L^{*}$. The action of $T_{X}$ on $E$-valued level forms is defined by

$$
T_{X}(\alpha \otimes e)=T_{X} \alpha \otimes e,
$$

for $e \in E$ and $\alpha \in \wedge^{j} L^{*}$. Since $m=\frac{1}{2} \operatorname{dim} L^{*}$, the operator $*_{l}: \wedge^{m} L^{*} \rightarrow \wedge^{m} L^{*}$ depends only on the conformal class $\left[g_{l}\right]$ of $g_{l}$. Thus the action of the operator $T_{X}$ on $\wedge^{m} L^{*}$ depends only on the data $\left(H, o_{l},\left[g_{l}\right]\right)$.

In this section, we suppose the $m$ is even. Then $*_{l}^{2}=1$ on $\wedge^{m} L^{*}$. Let $\wedge_{ \pm}^{m} L^{*}$ be the \pm 1 -eigenbundle of $*_{l}$ respectively. Then we have a basic orthogonal decomposition:

$$
\wedge^{m} L^{*}=\wedge_{+}^{m} L^{*} \oplus \wedge_{-}^{m} L^{*}
$$

Elements of $\wedge_{+}^{m} L^{*}$ and $\wedge_{-}^{m} L^{*}$ are called real self-dual level $m$-covectors and real anti-self-dual level $m$-covectors respectively. Let $\pi_{l}^{+}$and $\pi_{l}^{-}$denote the projections 
to the first and the second factor in (1.1) respectively. For any $\alpha \in \wedge^{m} L^{*}$, we have

$$
\alpha^{+} \stackrel{\text { def }}{=} \pi_{l}^{+} \alpha=\frac{\alpha+*_{l} \alpha}{2}, \quad \alpha^{-} \stackrel{\text { def }}{=} \pi_{l}^{-} \alpha=\frac{\alpha-*_{l} \alpha}{2} .
$$

The decomposition (1.1) induces

$$
\Omega_{l}^{m}(M)=\Omega_{+}^{m}(M) \oplus \Omega_{-}^{m}(M), \quad \Omega_{l}^{m}(E)=\Omega_{+}^{m}(E) \oplus \Omega_{-}^{m}(E),
$$

where $\Omega_{+}^{m}(M), \Omega_{-}^{m}(M)$ denote the spaces of sections of $\wedge_{+}^{m} L^{*}$ and $\wedge_{-}^{m} L^{*}$ respectively, and $\Omega_{+}^{m}(E), \Omega_{-}^{m}(E)$ denote the space of sections of $\wedge_{+}^{m} L^{*} \otimes_{\mathbb{R}} E$ and $\wedge_{-}^{m} L^{*} \otimes_{\mathbb{R}} E$ respectively.

For further constructions, we need a tangent codimension-one plane field $L$ which is transverse to the $H$. But we emphasize that in all sections except Section 8 in this paper, we do not need to assume $L$ is integrable: it can be any tangent smooth codimension-one plane field $L$. We now make the following convention throughout this paper.

Convention 1.1. $H$ is a smooth tangent line field over $M ; L^{*} \stackrel{\text { def }}{=} H^{\perp}$ is a smooth cotangent codimension-one plane field over $M ; o_{l}$ is an orientation on $L^{*} ; c_{l}$ is a conformal class of smooth positive definite metrics on $L^{*} ; g_{l}$ is a smooth positive definite metric on $L^{*} ; H^{*}$ is a smooth cotangent line field over $M$ such that $H^{*} \cap$ $L^{*}=\{0\} ; L \stackrel{\text { def }}{=} H^{* \perp}$ is a smooth tangent codimension-one plane field over $M$; $o$ is an orientation on $M ; g$ is a smooth Riemannian metric on $M$ with respect to which $H$ and $L$ are perpendicular; $o_{h}$ is an orientation on $H^{*}$ such that $o_{h} \wedge o_{l}=o$; and $g_{h}$ is a smooth positive definite metric on $H^{*}$ such that $g_{h} \oplus g_{l}=g$.

Remark 1.2. There are universal relations for these data: $L^{*}=H^{\perp}=H^{* \perp_{g}}$, $L=H^{\perp_{g}}=H^{* \perp}, g_{l}=\left.g\right|_{L^{*}}, g_{h}=\left.g\right|_{H^{*}}, g=g_{h} \oplus g_{l}, o=o_{h} \wedge o_{l}$. Here $\perp$ is with respect to the pairing of a vector and covector, and $\perp_{g}$ is with respect to the metric $g$. The sets of data $\left(H, o_{l}, c_{l}\right)$ and $\left(L^{*}, o_{l}, c_{l}\right)$ are equivalent. The sets of data $\left(H, o_{l}, g\right),\left(L^{*}, o_{l}, g\right),\left(L, o_{l}, g\right)$ and $\left(H^{*}, o_{l}, g\right)$ are all equivalent. The sets of data $\left(H, o, o_{l}, g\right),\left(H, o_{h}, o_{l}, g\right)$ and $\left(H, L, o_{l}, g_{l}, o_{h}, g_{h}\right)$ are equivalent. For the sake of brevity, a leaf of the foliation generated by a tangent line field $H$ is called a leaf of $H$.

Since $T^{*} M=H^{*} \oplus L^{*}$, we have direct sum decompositions

$$
\wedge^{j} T^{*} M=H^{*} \wedge \wedge^{j-1} L^{*} \oplus \wedge^{j} L^{*}, \quad \Omega^{j}(M)=\overline{H^{*}} \wedge \Omega_{l}^{j-1}(M) \oplus \Omega_{l}^{j}(M),
$$

where $\overline{H^{*}}$ is the space of sections of $H^{*}$ over $M$. Let $\pi_{h}$ denote the projection to the first factor, and let $\pi_{l}$ denote the projection to the second factor. For a form $\omega$ on $M$, we define

$$
\omega_{h} \stackrel{\text { def }}{=} \pi_{h} \omega \text { and } \omega_{l} \stackrel{\text { def }}{=} \pi_{l} \omega,
$$

which are called flow component and level component of $\omega$ respectively. Note $\pi_{h} f=0$ and $\pi_{l} f=f$ for any function $f$ on $M$. We have a basic decomposition of the exterior derivative $d$ on $M$ :

$$
\begin{gathered}
\qquad=d_{h}+d_{l}, \\
d_{h} \stackrel{\text { def }}{=} \pi_{h} d: \Omega^{j}(M) \rightarrow \overline{H^{*}} \wedge \Omega_{l}^{j-1}(M), \quad d_{l} \stackrel{\text { def }}{=} \pi_{l} d: \Omega^{j}(M) \rightarrow \Omega_{l}^{j}(M) .
\end{gathered}
$$

The decomposition (1.3) tensored with a vector bundle $E$ becomes:

$$
\Omega^{j}(E)=\overline{H^{*}} \wedge \Omega_{l}^{j-1}(E) \oplus \Omega_{l}^{j}(E) .
$$


Then we also have a unique decomposition of the covariant derivative $d_{A}$ of a connection $A$ on $E$ :

$$
\begin{gathered}
d_{A}=d_{A_{h}}+d_{A_{l}}, \\
d_{A_{h}} \stackrel{\text { def }}{=} \pi_{h} d_{A}: \Omega^{j}(E) \rightarrow \overline{H^{*}} \wedge \Omega_{l}^{j-1}(E), \quad d_{A_{l}} \stackrel{\text { def }}{=} \pi_{l} d_{A}: \Omega^{j}(E) \rightarrow \Omega_{l}^{j}(E) .
\end{gathered}
$$

The curvature $F_{A}$ decomposes uniquely as

$$
\begin{gathered}
F_{A}=F_{A_{h}}+F_{A_{l}}, \\
F_{A_{h}} \stackrel{\text { def }}{=} \pi_{h} F_{A} \in \overline{H^{*}} \wedge \Omega_{l}^{1}(E), \quad F_{A_{l}} \stackrel{\text { def }}{=} \pi_{l} F_{A} \in \Omega_{l}^{2}(E) .
\end{gathered}
$$

If $\tau$ is a section of $H^{*}$ such that $\langle\tau, X\rangle=1$ on an open subset $U$, then we have

$$
d_{h}=\tau \wedge \nabla_{X}, \quad d_{A_{h}}=\tau \wedge \nabla_{A_{X}}, \quad F_{A_{h}}=\tau \wedge F_{A_{X}} \quad \text { on } U .
$$

Using the decomposition (1.2), we define

$$
\begin{aligned}
& d_{A_{l}}^{+} \stackrel{\text { def }}{=} \pi_{l}^{+} d_{A_{l}}=\pi_{l}^{+} \pi_{l} d_{A}: \Omega^{m-1}(E) \rightarrow \Omega_{+}^{m}(E), \\
& d_{A_{l}}^{-} \stackrel{\text { def }}{=} \pi_{l}^{-} d_{A_{l}}=\pi_{l}^{-} \pi_{l} d_{A}: \Omega^{m-1}(E) \rightarrow \Omega_{-}^{m}(E),
\end{aligned}
$$

Now we define the most important spaces and operators in this paper:

$$
\begin{aligned}
\Omega_{++}^{m}(E) \stackrel{\text { def }}{=} \overline{H^{*}} & \wedge \Omega_{l}^{m-1}(E) \oplus \Omega_{+}^{m}(E), \quad \Omega_{+}^{m+1}(E) \stackrel{\text { def }}{=} \overline{H^{*}} \wedge \Omega_{+}^{m}(E), \\
& d_{A}^{++} \stackrel{\text { def }}{=} \pi^{++} d_{A}, \quad d_{A}^{++s} \stackrel{\text { def }}{=} l_{s} d_{A}^{++}, \\
& d_{A}^{+} \stackrel{\text { def }}{=} \pi^{+} d_{A}, \quad d_{A}^{+s} \stackrel{\text { def }}{=} d_{A}^{+} h_{s},
\end{aligned}
$$

where $\pi^{++}$denotes the projection from $\Omega^{m}(E)$ to $\Omega_{++}^{m}(E), \pi^{+}$denotes the projection from $\Omega^{m+1}(E)$ to $\Omega_{+}^{m+1}(E), s$ is a nonzero real or complex number, and $l_{s}, h_{s}$ are constant automorphisms of $\Omega_{++}^{m}(E)$ defined as:

$$
l_{s}(\theta+\alpha)=\theta+s \alpha, \quad h_{s}(\theta+\alpha)=s \theta+\alpha
$$

for $\theta \in \overline{H^{*}} \wedge \Omega_{l}^{m-1}(E)$ and $\alpha \in \Omega_{+}^{m}(M)$. Note the notational difference between the terms $\pi^{+}$and $\pi_{l}^{+}$. Plainly, we have

$$
\begin{gathered}
d_{A}=d_{A_{h}}+d_{A_{l}}=d_{A_{h}}+d_{A_{l}}^{+}+d_{A_{l}}^{-}=d_{A}^{++}+d_{A_{l}}^{-}, \\
l_{s} h_{s}=h_{s} l_{s}=s, \quad d_{A}^{+s} d_{A}^{++s}=s d_{A}^{+} d_{A}^{++} \text {on } \Omega_{++}^{m}(E) .
\end{gathered}
$$

Now we are ready to state our first main theorem.

Theorem 1.3. Let $M$ be a smooth $(2 m+1)$-manifold with even $m$ and with the data $\left(H, o_{l}, c_{l}\right)$ as in Convention 1.1. Assume that (1) $A$ is a flat connection on a real or complex vector bundle $E$ over $M$; (2) the level-star torsion operator $T_{X}=0$ on $d_{A_{l}}^{-}\left(\Omega_{l}^{m-1}(E)\right)$ for any $X \in H$. Then for any smooth tangent codimension-one plane field $L$ which is transverse to the $H$ and any nonzero real or complex number $s$, the following complex is well-defined and elliptic on $M$ :

$$
0 \rightarrow \Omega^{0}(E) \stackrel{d_{A}}{\rightarrow} \cdots \stackrel{d_{A}}{\rightarrow} \Omega^{m-1}(E) \stackrel{d_{A}^{++s}}{\rightarrow} \Omega_{++}^{m}(E) \stackrel{d_{A}^{+s}}{\rightarrow} \Omega_{+}^{m+1}(E) \rightarrow 0 .
$$

This complex is called a twisted half De Rham complex associated to the data $\left(H, o_{l}, c_{l}\right)$ and flat connection $A$. Theorem 1.3 will be proved in Section 3. The assumption on $T_{X}$ will be studied in Section 6 . When $o_{l}$ switches to its opposite $-o_{l}$, we have another elliptic complex by changing ++ to -- and + to - . 
Remark 1.4. We can put a Riemannian metric on $M$ and apply the Hodge star operator to the above complex, and get an elliptic complex which starts from the space of forms of the maximal degree $2 m+1$ to the corresponding space of forms of degree $m-1$ : the directions of the arrows are reversed. Therefore all the constructions and results in this paper for forms of degrees in the lower half range $\{0, \cdots, m+1\}$ can be transformed isomorphically by the Hodge star operator to forms of degrees in the upper half range $\{2 m+1, \cdots, m-1\}$.

The dimension 5 is special: twisted half De Rham complexes are well-defined and elliptic for twistings of a larger class of connections than the class of flat connections.

Definition 1.5. Let $M$ be a smooth 5 -manifold with the data $\left(H, o_{l}, c_{l}\right)$ as in Convention 1.1. A connection $A$ on a vector bundle $E$ over $M$ is called $\left(H, o_{l}, c_{l}\right)$ anti-self-dual if its curvature $F_{A}$ is a section of $\wedge_{-}^{2} L^{*} \otimes_{\mathbb{R}} \operatorname{Hom}(E, E)$, namely,

$$
F_{A} \in \Omega_{l}^{2}(\operatorname{Hom}(E, E)) \quad \text { and } \quad *_{l} F_{A}=-F_{A} .
$$

Here $E$ is either a real or a complex vector bundle; correspondingly, $\operatorname{Hom}(E, E)$ is either $\operatorname{Hom}_{\mathbb{R}}(E, E)$ or $\operatorname{Hom}_{\mathbb{C}}(E, E)$. A connection $A$ is called $\left(H, o_{l}, c_{l}\right)$-self-dual if it is $\left(H,-o_{l}, c_{l}\right)$-anti-self-dual, where $-o_{l}$ denotes the opposite orientation of $o_{l}$ on $L^{*}$.

By (1.4), a connection $A$ is $\left(H, o_{l}, c_{l}\right)$-anti-self-dual if and only if

$$
\pi_{l}^{+} F_{A_{l}}=0, \quad F_{A_{h}}=0,
$$

which is equivalent to

$$
d_{A}^{++} d_{A}=\pi^{++} F_{A}=F_{A_{h}}+\pi_{l}^{+} F_{A_{l}}=0 \quad \text { on } \Omega^{0}(E),
$$

for one (hence for all) tangent codimension-one plane field $L$ which is transverse to $H$.

Theorem 1.6. Assume that (1) $A$ is an $\left(H, o_{l}, c_{l}\right)$-anti-self-dual connection on a real or complex vector bundle $E$ over a smooth 5-manifold $M$; (2) the level-star torsion operator $T_{X}=0$ on $d_{A_{l}}^{-}\left(\Omega_{l}^{1}(E)\right)$ for $X \in H$. Then for any smooth tangent codimension-one plane field $L$ which is transverse to $H$ and any nonzero real or complex number $s$, the following complex is well-defined and elliptic on $M$ :

$$
0 \rightarrow \Omega^{0}(E) \stackrel{d_{A}}{\rightarrow} \Omega^{1}(E) \stackrel{d_{A}^{++s}}{\rightarrow} \Omega_{++}^{2}(E) \stackrel{d_{A}^{+s}}{\rightarrow} \Omega_{+}^{3}(E) \rightarrow 0 .
$$

The proof will be given in Section 3. The point is that (1.11) implies $d_{A}^{+s} d_{A}^{++s}=$ $s d_{A}^{+} d_{A}^{++}=0$ for all $s$ under the assumption on $T_{X}$. (See Proposition 3.4.)

Convention 1.7. In all the above notations as well as in Theorems 1.3 and 1.6, when $o_{l}$ switches to its opposite $-o_{l}$, we change the + signs to the - signs, the ++ signs to the -- signs, and "anti-self-dual" to "self-dual". When $d_{A}=d$, we change the term $E$ to $M$. This convention applies to notations in the next section too.

\section{Complex half De Rham complexes}

In this section we suppose that $m$ is an odd positive integer. Then $*_{l}^{2}=-1$ on $\wedge^{m} L^{*}$. Let $L_{c}^{*} \stackrel{\text { def }}{=} \mathbb{C} \otimes_{\mathbb{R}} L^{*}$. The level-star operator $*_{l}$ on $\wedge^{j} L^{*}$ extends complexlinearly on $\wedge^{j} L_{c}^{*} \stackrel{\text { def }}{=} \mathbb{C} \otimes_{\mathbb{R}} \wedge^{j} L^{*}$ for all $j$. Note $L_{c}^{*}=\mathbb{C} \otimes_{\mathbb{R}} L^{*}$ has an obvious complex structure $J$ given by $J \alpha=i \otimes \alpha$ and $J(i \otimes \alpha)=-\alpha$ for any $\alpha \in L^{*}$. The Hermitian 
level-metric $g_{l}^{c}$ on $L_{c}^{*}$ is defined by $\alpha \wedge *_{l} \bar{\beta}=g_{l}^{c}(\alpha, \beta) \operatorname{vol}\left(g_{l}\right)$, where $\operatorname{vol}\left(g_{l}\right)$ is the oriented volume form of $g_{l}$ and $\bar{\beta}$ is the complex conjugate of $\beta$ with respect to the complex structure $J$. Since $*_{l}$ extends complex-linearly, we still have $*_{l}^{2}=-1$ on $\wedge^{m} L_{c}^{*}$, and we get

$$
\wedge^{m} L_{c}^{*}=\wedge_{+}^{m} L_{c}^{*} \oplus \wedge_{-}^{m} L_{c}^{*},
$$

where $\wedge_{ \pm}^{m} L_{c}^{*}$ are the $\pm i$ eigenbundles of $*_{l}$ respectively. Elements of $\wedge_{+}^{m} L_{c}^{*}$ and $\wedge_{-}^{m} L_{c}^{*}$ are called level self-dual complex $m$-covectors and level anti-self-dual complex $m$-covectors respectively. Still let $\pi_{l}^{+}$and $\pi_{l}^{-}$denote the projections to the first and the second factor of (2.1) respectively. Plainly, for any $\alpha \in \wedge^{m} L_{c}^{*}$, we have

$$
\alpha^{+} \stackrel{\text { def }}{=} \pi_{l}^{+} \alpha=\frac{\alpha-i *_{l} \alpha}{2}, \quad \alpha^{-} \stackrel{\text { def }}{=} \pi_{l}^{-} \alpha=\frac{\alpha+i *_{l} \alpha}{2} .
$$

For a complex vector bundle $E$, let $\Omega_{ \pm}^{m}(E)$ denote the space of smooth sections of $\wedge_{ \pm}^{m} L_{c}^{*} \otimes_{\mathbb{R}} E$. Then we have all the corresponding constructions as those in the last section. To avoid too many notations, we still use the terms $\pi_{l}^{ \pm}, \pi^{++}, \pi^{--}$, $\pi^{ \pm}, d_{A}^{++s}, d_{A}^{+s}, \Omega_{++}^{m}(E)$ and $\Omega_{+}^{m+1}(E)$, which have the corresponding meanings in this complex context.

The complex structure of a complex vector bundle $E$ induces a canonical complex structure on $\wedge^{j} T^{*} M \otimes_{\mathbb{R}} E$ by $i\left(\omega \otimes_{\mathbb{R}} e\right) \stackrel{\text { def }}{=} \omega \otimes_{\mathbb{R}} i e$. We have canonically identifications: $i\left(\omega \otimes_{\mathbb{R}} e\right)=i\left(\omega \otimes_{\mathbb{C}} e\right)=i \omega \otimes_{\mathbb{C}} e=\omega \otimes_{\mathbb{C}} i e, \wedge^{j} T^{*} M \otimes_{\mathbb{R}} E=\wedge^{j} T_{c}^{*} M \otimes_{\mathbb{C}} E$ and $\wedge^{j} L^{*} \otimes_{\mathbb{R}} E=\wedge^{j} L_{c}^{*} \otimes_{\mathbb{C}} E$, where $T_{c}^{*} M \stackrel{\text { def }}{=} \mathbb{C} \otimes_{\mathbb{R}} T^{*} M$.

In order to state results in this paper in a uniform manner for all positive integers $m$, despite of the parity of $m$, we need to make another notational convention.

Convention 2.1. When $m$ is odd, all forms are complex forms; $\Omega^{j}(M)$ means the space of sections of $\wedge^{j} T_{c}^{*} M ; \Omega_{l}^{j}(M)$ means the space of sections of $\wedge^{j} L_{c}^{*}$; $E$ means a complex vector bundle; $\Omega^{j}(E)$ means the space of sections of $\wedge^{j} T_{c}^{*} M \otimes_{\mathbb{C}} E ; \Omega_{l}^{j}(E)$ means the space of sections of $\wedge^{j} L_{c}^{*} \otimes_{\mathbb{C}} E ; H^{j}(M)$ means $H^{j}(M, \mathbb{C}) ; \operatorname{dim}$ means complex dimension; and $s$ is a nonzero complex number.

When $m$ is even, all forms are real forms; $\Omega^{j}(M)$ means the space of sections of $\wedge^{j} T^{*} M ; \Omega_{l}^{j}(M)$ means the space of sections of $\wedge^{j} L^{*} ; E$ means a real or complex vector bundle; $\Omega^{j}(E)$ means the space of sections of $\wedge^{j} T^{*} M \otimes_{\mathbb{R}} E ; \Omega_{l}^{j}(E)$ means the space of sections of $\wedge^{j} L^{*} \otimes_{\mathbb{R}} E ; H^{j}(M)$ means $H^{j}(M, \mathbb{R})$; dim means real dimensions; and $s$ is a nonzero real (resp. complex) number if $d_{A}^{++s}$ acts on a real (resp. complex) vector bundle.

In notations like $\operatorname{dim} \mathcal{H}_{+}^{m}(A)$, the term $A$ means the pair $(A, E)$. In the literature, the same term $A$ is often used to denote all induced connections on all the bundles associated to $E$. In that case, for the meaning of $\operatorname{dim} \mathcal{H}_{+}^{m}(A)$ one needs to specify which bundle $E$ is referred to.

Theorem 2.2. Let $M$ be a smooth $(2 m+1)$-manifold with odd $m$ and with the data $\left(H, o_{l}, c_{l}\right)$ as in Convention 1.1. Assume that (1) $A$ is a flat connection on a complex vector bundle $E$ over $M$; (2) the level-star torsion operator $T_{X}=0$ on $d_{A_{l}}^{-}\left(\Omega_{l}^{m-1}(E)\right)$ for any $X \in H$. Then for any smooth tangent codimension-one plane field $L$ which is transverse to $H$ and any nonzero complex number $s$, the following complex is well-defined and elliptic on $M$ :

$$
0 \rightarrow \Omega^{0}(E) \stackrel{d_{A}}{\rightarrow} \cdots \stackrel{d_{A}}{\rightarrow} \Omega^{m-1}(E) \stackrel{d_{A}^{++s}}{\rightarrow} \Omega_{++}^{m}(E) \stackrel{d_{A}^{+s}}{\rightarrow} \Omega_{+}^{m+1}(E) \rightarrow 0 .
$$


This complex is called a complex twisted half De Rham complex associated to the data $\left(H, o_{l}, c_{l}\right)$ and flat connection $A$. The proof will be given in Section 3.

The dimension 3 is special: twisted half De Rham complexes are well-defined and elliptic for twistings of a larger class of connections than the class of flat connections.

Definition 2.3. Let $M$ be a smooth 3 -manifold with the data $\left(H, o_{l}, c_{l}\right)$ as in Convention 1.1. A connection $A$ on a complex vector bundle $E$ over $M$ is called $\left(H, o_{l}, c_{l}\right)$-anti-self-dual if $F_{A_{X}} \stackrel{\text { def }}{=} i_{X} F_{A}$ is a section of $\wedge_{-}^{1} L_{c}^{*} \otimes_{\mathbb{C}} H o m_{\mathbb{C}}(E, E)$ for any section $X$ of $H$, that is,

$$
*_{l} F_{A_{X}}=-i F_{A_{X}} .
$$

A connection $A$ is called $\left(H,-o_{l}, c_{l}\right)$-self-dual if it is $\left(H,-o_{l}, c_{l}\right)$-anti-self-dual. By (1.5), $A$ is $\left(H, o_{l}, c_{l}\right)$-anti-self-dual if and only if

$$
\pi^{+} F_{A_{h}}=0
$$

for one (hence for all) tangent codimension-one plane field $L$ which is transverse to $H$. If $T_{X}=0$ on $\wedge_{-}^{1} L_{c}^{*}$, then (2.3) is equivalent to

$$
d_{A}^{+} d_{A}^{++}=0 \text { on } \Omega^{0}(E)
$$

according to Proposition 3.4.

Theorem 2.4. Assume that (1) $A$ is an $\left(H, o_{l}, c_{l}\right)$-anti-self-dual connection on a complex vector bundle $E$ over a smooth 3-manifold $M$; and (2) the level-star torsion operator $T_{X}=0$ on $d_{A_{l}}^{-}\left(\Omega^{0}(E)\right)$ for $X \in H$. Then for any smooth tangent codimension-one plane field $L$ which is transverse to $H$ and for any nonzero complex number s, the following complex is well-defined and elliptic on $M$ :

$$
0 \rightarrow \Omega^{0}(E) \stackrel{d_{A}^{++s}}{\rightarrow} \Omega_{++}^{1}(E) \stackrel{d_{A}^{+s}}{\rightarrow} \Omega_{+}^{2}(E) \rightarrow 0 .
$$

The proof will be given in the next section.

\section{Proof OF WELL-DEFINEDNESS AND ELLIPTICITY}

In this section, we present the proofs that the complexes in Theorems 1.3, 1.6, 2.2 and 2.4 are well-defined and elliptic. First of all, we need some basic formulas. In the following three lemmas, $A$ is a connection on a vector bundle $E$ over $M$, $\omega$ is an $E$-valued $j$-form, $\alpha$ is an $E$-valued level $j$-form, $\beta$ is an $E$-valued level $(j-1)$-form, $\gamma$ is a level form, $f$ is a function, and $e$ is a section of $E$.

Lemma 3.1. Let $M$ be a smooth n-manifold with a smooth tangent line field $H$. Let $X$ be a section of $H$ over $M$. Then

(1) $\nabla_{A_{X}} \omega$ is always an E-valued level form, and $\nabla_{X}=L_{X}$ on level forms.

(2) $\nabla_{A_{X}}(\gamma \wedge \alpha)=\nabla_{X} \gamma \wedge \alpha+\gamma \wedge \nabla_{A_{X}} \alpha$.

Lemma 3.2. Let $M$ be a smooth $(2 m+1)$-manifold with the data $\left(H, o_{l}, g_{l}\right)$ as in Convention 1.1. Let $X \in H, \epsilon=1$ for even $m$ and $\epsilon=-i$ for odd $m$. Then

(1) $T_{X}(f \gamma)=f T_{X} \gamma$ and $T_{f X}=f T_{X}$

(2) $T_{X}(\gamma \otimes e)=\left(\nabla_{A_{X}} *_{l}-*_{l} \nabla_{A_{X}}\right)(\gamma \otimes e)$ and $\left[\nabla_{A_{X}}, \pi_{l}^{+}\right]=\frac{\epsilon}{2} T_{X}=\left[\pi_{l}^{-}, \nabla_{A_{X}}\right]$;

(3) $T_{X} *_{l} \alpha=-*_{l} T_{X} \alpha, T_{X} \pi_{l}^{+}=\pi_{l}^{-} T_{X}$ and $T_{X} \pi_{l}^{-}=\pi_{l}^{+} T_{X}$ on $\wedge^{m} L^{*} \otimes E$.

Lemma 3.3. Let $M$ be a smooth n-manifold with the data $(H, L)$ as in Convention 1.1. Let $X$ be any section of $H$ over an open subset $U$ and $\tau$ be any section of $H^{*}$ over $U$ such that $\langle X, \tau\rangle=1$ on $U$. Then 
(1) $d_{A} \omega=\tau \wedge \nabla_{A_{X}} \omega+d_{A_{l}} \omega$ and $d_{A} \alpha=\tau \wedge \nabla_{A_{X}} \alpha+d_{A_{l}} \alpha$ on $U$.

(2) $d_{A}(\tau \wedge \beta)=-\tau \wedge d_{A_{l}} \beta+d \tau \wedge \beta=\tau \wedge\left[-d_{A_{l}} \beta+\left(\nabla_{X} \tau\right) \beta\right]+d_{l} \tau \wedge \beta$ on $U$.

(3) $d_{A_{l}}(\gamma \wedge \alpha)=d_{l} \gamma \wedge \alpha+(-1)^{\operatorname{deg} \gamma} \gamma \wedge d_{A_{l}} \alpha$ on $U$.

(4) $d_{A_{h}}(\gamma \wedge \alpha)=d_{h} \gamma \wedge \alpha+(-1)^{\operatorname{deg} \gamma} \gamma \wedge d_{A_{h}} \alpha$ on $U$. $\beta$.

Note that $\nabla_{X} \tau$ is a function on $U$, and the term $d \tau \wedge \beta$ is algebraically linear in

In showing that the half De Rham complexes are well-defined, we present the main calculation as follows.

Proposition 3.4. Let $M$ be a smooth $(2 m+1)$-manifold with the data $\left(H, o_{l}, g_{l}, L\right)$ as in Convention 1.1, where $m \geq 1$. Let $A$ be a connection on a real or complex vector bundle $E, X$ be a section of $H$ over an open susbet $U$ of $M$, and $\tau$ be a section of $H^{*}$ over $U$ such that $\langle\tau, X\rangle=1$. Then

$$
d_{A}^{+s} d_{A}^{++s} \omega=s \tau \wedge\left[\frac{\epsilon}{2} T_{X} d_{A_{l}}^{-} \omega+\pi_{l}^{+}\left(F_{A_{X}} \wedge \alpha\right)+\pi_{l}^{+}\left(F_{A_{l}} \wedge \beta\right)\right]
$$

for all $\omega \in \Omega^{m-1}\left(\left.E\right|_{U}\right)$, where $\omega=\tau \wedge \beta+\alpha$ with $\beta \in \Omega_{l}^{m-2}\left(\left.E\right|_{U}\right)$ and $\alpha \in$ $\Omega_{l}^{m-1}\left(\left.E\right|_{U}\right), \epsilon=1$ for even $m$ and $\epsilon=-i$ for odd $m$.

Remark 3.5. In the case $m=1, \pi_{l}^{+} F_{A_{l}} \wedge \beta=0$ always holds because $\beta \in$ $\Omega_{l}^{m-2}\left(\left.E\right|_{U}\right)=0$, and $\pi_{l}^{+}\left(F_{A_{X}} \wedge \alpha\right)=\left(\pi_{l}^{+} F_{A_{X}}\right) \alpha=i_{X}\left(\pi^{+} F_{A_{h}}\right) \alpha$, because $\alpha$ is a section of $\left.E\right|_{U}$ over $U$. In the case $m=2, \pi_{l}^{+}\left(F_{A_{l}} \wedge \beta\right)=\left(\pi_{l}^{+} F_{A_{l}}\right) \beta$, since this $\beta$ is a section of $\left.E\right|_{U}$ over $U$.

Proof of Proposition 3.4. Since $d_{A}^{+s} d_{A}^{++s} \omega=s d_{A}^{+} d_{A}^{++}$for all $s$, we only need to prove (3.1) in the case $s=1$. Recall that $F_{A}=\tau \wedge F_{A_{X}}+F_{A_{l}}$. Using the decomposition (1.7), formula (1) of Lemma 3.3 and formula (2) of Lemma 3.2, we have

$$
\begin{aligned}
& d_{A}^{+} d_{A}^{++} \omega=\pi^{+} d_{A}\left(d_{A}-d_{A_{l}}^{-}\right) \omega \\
& =\pi^{+}\left(F_{A} \wedge \omega\right)-\pi^{+} d_{A} d_{A_{l}}^{-} \omega \\
& =\pi^{+}\left(F_{A} \wedge \omega\right)-\pi^{+}\left(\tau \wedge \nabla_{A_{X}} d_{A_{l}}^{-} \omega+d_{A_{l}} d_{A_{l}}^{-} \omega\right) \\
& =\pi^{+}\left[\left(\tau \wedge F_{A_{X}}+F_{A_{l}}\right) \wedge(\tau \wedge \beta+\alpha)\right]-\tau \wedge \pi_{l}^{+} \nabla_{A_{X}} d_{A_{l}}^{-} \omega \\
& =\tau \wedge \pi_{l}^{+}\left(F_{A_{X}} \wedge \alpha+F_{A_{l}} \wedge \beta\right)-\tau \wedge \nabla_{A_{X}} \pi_{l}^{+} d_{A_{l}}^{-} \omega+\tau \wedge \frac{\epsilon}{2} T_{X} d_{A_{l}}^{-} \omega \\
& =\tau \wedge\left[\frac{\epsilon}{2} T_{X} d_{A_{l}}^{-} \omega+\pi_{l}^{+}\left(F_{A_{X}} \wedge \alpha\right)+\pi_{l}^{+}\left(F_{A_{l}} \wedge \beta\right)\right] .
\end{aligned}
$$

Note that in the above computations, we have used $\pi^{+}\left(d_{A_{l}} d_{A_{l}}^{-} \omega\right)=0$, which follows from the definition of $\pi^{+}$, and used $\pi_{l}^{+} d_{A_{l}}^{-} \omega=\pi_{l}^{+} \pi_{l}^{-} d_{A_{l}} \omega=0$, which follows from the definitions of $\pi_{l}^{+}$and $\pi_{l}^{-}$.

Lemma 3.6. Let $T$ denote a fiber of the $L^{*}$ in the case $m$ is even, and a fiber of the $L_{c}^{*}$ in the case $m$ is odd. Then $\pi_{l}^{+}: \rho \wedge\left(\wedge^{m-1} T\right) \rightarrow \wedge_{+}^{m} T$, which sends $\rho \wedge \alpha$ to $\pi_{l}^{+}(\rho \wedge \alpha)$, is bijective for any nonzero vector $\rho \in T$, where $\alpha \in \wedge^{m-1} T$.

Proof. Since $\operatorname{dim} \wedge_{+}^{m} T=\operatorname{dim} \rho \wedge\left(\wedge^{m-1} T\right)$, we need only to prove the injectivity. Expand $\rho$ to an oriented orthogonal real basis of the same length for $T: \rho^{1}=\rho$, $\rho^{2}, \ldots, \rho^{2 m}$. Let $J$ be an ordered subset of $\{1, \ldots, 2 m\},|J|$ the length of $J$, and let $\rho^{J}$ be the ordered wedge product of $\rho^{j}$ for $j \in J . \rho^{1} \wedge \wedge^{m-1} T$ has a basis $\left\{\rho^{1 J}\right.$ : $1 \notin J$ and $|J|=m-1\}$. In the case $m$ is even, $\wedge_{ \pm}^{m} T$ has a basis $\left\{\rho^{1 J} \pm *_{l} \rho^{1 J}\right.$ : 
$1 \notin J$ and $|J|=m-1\}$. Thus any element $\omega \in \rho^{1} \wedge\left(\wedge^{m-1} T\right)$ can be written as $\sum_{J} x_{J} \rho^{1} \wedge \rho^{J}$, where $x_{J}$ is a number. Note that $\pi_{l}^{+} \omega=\sum_{J} x_{J} \frac{1}{2}\left(\rho^{1} \wedge \rho^{J}+*_{l} \rho^{1 J}\right)$. Hence if $\pi_{l}^{+} \omega=0$ then all $x_{J}=0$, i.e., $\omega=0$. In the case $m$ is odd, using a basis $\left\{\rho^{1 J} \mp i *_{l} \rho^{1 J}: 1 \notin J\right.$ and $\left.|J|=m-1\right\}$ for $\wedge_{ \pm}^{m} T$, we can prove the statement in the same way.

Proof of Theorems 1.3, 1.6, 2.2 and 2.4. When $\operatorname{dim} M=3, d_{A}^{+s} d_{A}^{++s}=0$ follows from (2.3), the assumption on $T_{X}$, Proposition 3.4 and Remark 3.5.

When $\operatorname{dim} M=5, d_{A}^{+s} d_{A}^{++s}=0$ follows from (1.10), the assumption on $T_{X}$, Proposition 3.4 and Remark 3.5. Moreover (1.10) yields $d_{A}^{++s} d_{A}=l_{s} \pi^{++} d_{A}^{2}=$ $l_{s} \circ\left(F_{A_{h}}+\pi_{l}^{+} F_{A_{l}}\right)=0$.

When $\operatorname{dim} M>5$, we have $d_{A}^{2}=0$ and $d_{A}^{++s} d_{A}=l_{s} \pi^{++} d_{A}^{2}=0$. Then Proposition 3.4 and the assumption on $T_{X}$ together yield $d_{A}^{+s} d_{A}^{++s}=0$.

Now we are about to prove ellipticity of the half De Rham complex in the case $m$ is even. The ellipticity in the case $m$ is odd can be proved in the same way, based on the complex part of Lemma 3.6. Fix an arbitrary point $x$ in $M$. In this proof, the terms $H^{*}, L^{*}, \wedge^{j} T^{*} M, \wedge^{j} L^{*}$ and $\wedge_{+}^{m} L^{*}$ denote respectively the fiber of bundles $H^{*}, L^{*}, \wedge^{j} T^{*} M, \wedge^{j} L^{*}$ and $\wedge_{+}^{m} L^{*}$ at $x$. Let $\xi=k \tau+\rho \neq 0$ be a nonzero covector at $x$, where $k$ is a real number, $\tau \in H^{*}$ and $\rho \in L^{*}$.

The symbol sequence of a half De Rham complex at $\xi$ is

$$
\begin{aligned}
0 \rightarrow \mathbb{R} \stackrel{\sigma}{\rightarrow} T^{*} M & \rightarrow \\
\rightarrow & \stackrel{\sigma}{\rightarrow} \wedge^{m-1} T^{*} M \stackrel{l_{s} \sigma^{++}}{\rightarrow} \tau \wedge \wedge^{m-1} L^{*} \oplus \wedge_{+}^{m} L^{*} \\
\stackrel{\sigma^{+} h_{s}}{\rightarrow} & \wedge \wedge_{+}^{m} L^{*} \rightarrow 0,
\end{aligned}
$$

where $\sigma, \sigma^{++}, \sigma^{+}$denote $-i \sigma_{\xi}(d),-i \sigma_{\xi}\left(d^{++}\right)$and $-i \sigma_{\xi}\left(d^{+}\right)$respectively. Since the principal symbol sequence of the complex twisted by a connection $A$ is just (3.2) tensored with $E$, we need only to give the proof in the case $d_{A}=d$. Further, because the sequence (3.2) before the last three stages is the same as the symbol sequence of the usual De Rham complex, and because $l_{s}, h_{s}$ are automorphisms, we need only to prove the exactness at the last three stages of (3.2) for $s=1$. Note that

$$
\begin{aligned}
& \sigma(\omega)=\xi \wedge \omega, \\
& \sigma^{++}(\tau \wedge \beta+\alpha)=\pi^{++}[\xi \wedge(\tau \wedge \beta+\alpha)]=\tau \wedge(k \alpha-\rho \wedge \beta)+\pi_{l}^{+}(\rho \wedge \alpha), \\
& \sigma^{+}(\tau \wedge \alpha+\gamma)=\pi^{+}[\xi \wedge(\tau \wedge \alpha+\gamma)]=\tau \wedge\left[k \gamma-\pi_{l}^{+}(\rho \wedge \alpha)\right],
\end{aligned}
$$

for a level $(m-2)$-covector $\beta$, a level $(m-1)$-covector $\alpha$ and a level self-dual $m$-covector $\gamma$.

Step 1. To prove $K e r \sigma^{++}=\operatorname{Im} \sigma$.

Let $\omega=\tau \wedge \beta+\alpha$, where $\beta \in \wedge^{m-2} L^{*}, \alpha \in \wedge^{m-1} L^{*}$. If $\sigma^{++}(\tau \wedge \beta+\alpha)=$ $\tau \wedge(k \alpha-\rho \wedge \beta)+\pi_{l}^{+}(\rho \wedge \alpha)=0$, then $k \alpha-\rho \wedge \beta=0$ and $\pi_{l}^{+}(\rho \wedge \alpha)=0$.

Case 1.1. Suppose $\rho=0$. Then $k \alpha-\rho \wedge \beta=0$ means $\alpha=0$, and $k$ has to be nonzero. Thus $\omega=\tau \wedge \beta=\sigma\left(\frac{\beta}{k}\right)$ is in the image of $\sigma$.

Case 1.2. Suppose $\rho \neq 0$. Then by the injectivity in Lemma 3.6, $\pi_{l}^{+}(\rho \wedge \alpha)=0$ implies $\rho \wedge \alpha=0$. Thus $\xi \wedge(\tau \wedge \beta+\alpha)=\tau \wedge(k \alpha-\rho \wedge \beta)+\rho \wedge \alpha=0$. Since $\xi \neq 0$, $\tau \wedge \beta+\alpha=\xi \wedge$ (some $(m-2)$-covector), which is in the image of $\sigma$.

Step 2. To prove $K e r \sigma^{+}=I m \sigma^{++}$.

Let $\omega=\tau \wedge \alpha+\gamma$, where $\alpha \in \wedge^{m-1} L^{*}, \gamma \in \wedge^{m} L^{*}$. If $\sigma^{+} \omega=\sigma^{+}(\tau \alpha+\gamma)=$ $\tau \wedge\left[k \gamma-\pi_{l}^{+}(\rho \wedge \alpha)\right]=0$, then $k \gamma=\pi_{l}^{+}(\rho \wedge \alpha)$. 
Case 2.1. Suppose $\rho=0$. Then $k \neq 0$. Thus $\gamma=0$, and so $\tau \wedge \alpha+\gamma=\sigma^{++}\left(\frac{\alpha}{k}\right)$ is in the image of $\sigma^{++}$.

Case 2.2. Suppose $\rho \neq 0$ and $k \neq 0$. Then $\rho$ can be extended as a linear basis of $L^{*}: \rho^{1}=\rho, \rho^{2}, \ldots, \rho^{2 m}$. Any $\alpha \in \wedge^{m-1} L^{*}$ can be expressed as $\alpha=\rho \wedge \alpha^{\prime}+\alpha^{\prime \prime}$, where $\alpha^{\prime}$ and $\alpha^{\prime \prime}$ can be expressed in terms of $\rho_{2}, \ldots, \rho_{2 m}$. Hence $\tau \wedge \alpha+\gamma=$ $\tau \wedge \alpha+\frac{1}{k} \pi_{l}^{+}(\rho \wedge \alpha)=\tau \wedge\left(\alpha^{\prime \prime}+\rho \wedge \alpha^{\prime}\right)+\pi_{l}^{+}\left(\rho \wedge \frac{\alpha^{\prime \prime}}{k}\right)=\sigma^{++}\left(-\tau \wedge \alpha^{\prime}+\frac{\alpha^{\prime \prime}}{k}\right)$ is in the image of $\sigma^{++}$.

Case 2.3. Suppose $\rho \neq 0$ and $k=0$. Then $\pi_{l}^{+}(\rho \wedge \alpha)=0$. The injectivity in Lemma 3.6 implies $\rho \wedge \alpha=0$. Thus $\alpha=\rho \wedge \alpha^{\prime}$ for some level $(m-2)$-covector $\alpha^{\prime}$. By surjectivity in Lemma 3.6, we have $\gamma=\pi_{l}^{+}\left(\rho \wedge \gamma^{\prime}\right)$ for some level $(m-1)$-covector $\gamma^{\prime}$. Hence $\tau \wedge \alpha+\gamma=\tau \wedge\left(\rho \wedge \gamma^{\prime}\right)+\pi_{l}^{+}\left(\rho \wedge \gamma^{\prime}\right)=\sigma^{++}\left(-\tau \wedge \alpha^{\prime}+\gamma^{\prime}\right)$ is in the image of $\sigma^{++}$.

Step 3. To prove $\sigma^{+}$is surjective.

Case 3.1. If $k \neq 0$, then $\tau \wedge \gamma=\sigma^{+}\left(\frac{\gamma}{k}\right)$ is in the image of $\sigma^{+}$.

Case 3.2. $k=0$ and $\rho \neq 0$. Then by the surjectivity in Lemma 3.6, any $\gamma=\pi_{l}^{+}\left(\rho \wedge \gamma^{\prime}\right)$ for some level $(m-1)$-covector $\gamma^{\prime}$. Thus $\tau \wedge \gamma=\tau \wedge \pi_{l}^{+}\left(\rho \wedge \gamma^{\prime}\right)$ $=\sigma^{+}\left(\tau \wedge\left(-\gamma^{\prime}\right)\right)$ is in the image of $\sigma^{+}$.

\section{ON CLOSED ODD-DIMENSIONAL MANIFOLDS}

In this section, we study half De Rham complexes on closed manifolds. Firstly let us make a definition.

Definition 4.1. Let $M$ be a closed smooth $(2 m+1)$-manifold with the data $\left(H, o_{l}, c_{l}, L\right)$ as in Convention 1.1. When the complex in Theorems 1.3, 1.6, 2.2 and 2.4 is well-defined, its cohomology groups are denoted by $H^{j}(A), H_{+}^{m-1}(A)$, $H_{+s}^{m}(A)$ and $H_{+}^{m+1}(A)$, where $0 \leq j \leq m-2$. They are called self-duality cohomology groups.

When $d_{A}=d$, the exterior derivative on $M$, we replace the term $A$ by $M$ in the above notations.

Remark 4.2. Since $\operatorname{Kerd}_{A}^{++s}=\operatorname{Kerd}_{A}^{++}$and $d_{A}^{+s}\left(\Omega_{++}^{m}(E)\right)=d_{A}^{+}\left(\Omega_{++}^{m}(E)\right)$, all self-duality cohomology groups except $H_{+s}^{m}(A)$ are independent of $s$. For $H_{+s}^{m}(A)$, we have $h_{s}\left(H_{+s}^{m}(A)\right)=H_{+1}^{m}(A)$, where $h_{s}$ maps $[\omega]$ to $\left[h_{s} \omega\right]$ with the inverse $h_{s}^{-1}=$ $s^{-1} l_{s}$.

Let $E$ be equipped with a Riemannian or Hermitian metric. In the following formulas, when the metric on $E$ is Riemannian, we regard the conjugation operation as the identity map. Define $(\alpha \otimes e) \wedge(\beta \otimes \bar{f})=\alpha \wedge \beta(e, f)$ for forms $\alpha, \beta$ on $M$ and sections $e, f$ of $E$. Then $\langle\omega, \eta\rangle \operatorname{vol}(g)=\omega \wedge * \bar{\eta}$ for all $E$-valued forms $\omega$ and $\eta$. The formal adjoint operator $d_{A}^{*}$ of $d_{A}$ is defined by

$$
\left(\omega, d_{A}^{*} \eta\right) \stackrel{\text { def }}{=}\left(d_{A} \omega, \eta\right)
$$

where $\omega$ is an $E$-valued ( $j-1)$-form compactly supported in the interior of $M, \eta$ is an $E$-valued $j$-form, and $(\omega, \eta) \stackrel{\text { def }}{=} \int_{M}\langle\omega, \eta\rangle \operatorname{vol}(g)$. The formal adjoint operator $\left(d_{A}^{++s}\right)^{*}$ of $d_{A}^{++s}$ and the formal adjoint operator $\left(d_{A}^{+s}\right)^{*}$ of $d_{A}^{+s}$ are defined similarly by (4.1): we just replace $d_{A}$ by $d_{A}^{++s}$ and $d_{A}^{+s}$ respectively. It is easy to check that

$$
\left(d_{A}^{++s}\right)^{*}=d_{A}^{*} l_{\bar{s}}, \quad\left(d_{A}^{+s}\right)^{*}=h_{\bar{s}} \pi^{++} d_{A}^{*},
$$

where $\bar{s}$ denotes the complex conjugate of $s$. 
If the metric on $E$ is $d_{A}$-parallel-invariant, then

$$
d(e, f)=\left(d_{A} e, f\right)+\left(e, d_{A} f\right), \quad d(\omega \wedge \bar{\eta})=d_{A} \omega \wedge \eta+(-1)^{\operatorname{deg} \omega} \omega \wedge d_{A} \bar{\eta}
$$

for sections $e, f$ of $E$ and $E$-valued forms $\omega, \eta$. These identities together with (4.1) give

$$
d_{A}^{*} \eta=(-1)^{\operatorname{deg} \eta} *^{-1} d_{A} * \eta .
$$

Recall from Remark 1.2 that the data $\left(H, o_{l}, o, g\right)$ on $M$ induces data

$$
\left(H, L, o_{l}, g_{l}, o_{h}, g_{h}\right) \text {. }
$$

The oriented volume form $\operatorname{vol}(g)$ of $g$ and the level-oriented volume form $\operatorname{vol}\left(g_{l}\right)$ of $g_{l}$ are related by $\operatorname{vol}(g)=\frac{\tau}{|\tau|} \wedge \operatorname{vol}\left(g_{l}\right)$, where $\tau$ is a nonvanishing section of $H^{*}$ defining the $o_{h}$, and $|\tau|$ is the length of $\tau$ with respect to the $g_{h}$. Let $*$ denote the star operator of the metric $g$ on $M$. It is easy to check the following formulas.

Lemma 4.3. Let $M$ be an $n$-manifold with the data $\left(H, o_{l}, o, g\right)$ as in Convention 1.1. Let $\alpha$ be an E-valued level $j$-form, $\beta$ be an E-valued level $(j-1)$-form, $X$ be a section of $H$ over an open subset $U$ and $\tau$ be a section of $H^{*}$ over $U$ such that $\langle X, \tau\rangle=1$ on $U$. Then

$$
\begin{gathered}
*(\tau \wedge \beta+\alpha)=(-1)^{j} \tau \wedge \frac{1}{|\tau|} *_{l} \alpha+|\tau| *_{l} \beta, \\
d_{A} *(\tau \wedge \beta)=\tau \wedge \nabla_{A_{X}}\left(|\tau| *_{l} \beta\right)+d_{A_{l}}|\tau| *_{l} \beta, \\
* d_{A} *(\tau \wedge \beta)=(-1)^{n-j+1} \tau \wedge \frac{1}{|\tau|} *_{l} d_{A_{l}}|\tau| *_{l} \beta+|\tau| *_{l} \nabla_{A_{X}}\left(|\tau| *_{l} \beta\right) .
\end{gathered}
$$

Definition 4.4. Suppose $M$ is an oriented closed smooth $(2 m+1)$-manifold with the data $\left(H, o_{l}, o, g\right)$ as in Convention 1.1. Let $0 \leq j \leq m-2$. Define

$$
\begin{aligned}
& \mathcal{H}_{+}^{m+1}(A) \stackrel{\text { def }}{=} \operatorname{Ker}\left(d_{A}^{+}\right)^{*}=\operatorname{Ker}\left(d_{A}^{+s}\right)^{*}, \\
& \mathcal{H}_{+s}^{m}(A) \stackrel{\text { def }}{=} \operatorname{Kerd} d_{A}^{+s} \cap\left(d_{A}^{++s}\right)^{*}, \\
& \mathcal{H}_{+}^{m-1}(A) \stackrel{\text { def }}{=} \operatorname{Kerd} d_{A}^{++} \cap K \operatorname{Ker} d_{A}^{*}=\operatorname{Kerd}_{A}^{++s} \cap \operatorname{Kerd} d_{A}^{*}, \\
& \mathcal{H}^{j}(A) \stackrel{\text { def }}{=} \operatorname{Kerd} d_{A} \cap K \operatorname{Kerd} d_{A}^{*}, \\
& b_{+}^{m+1}(A) \stackrel{\text { def }}{=} \operatorname{dim} \mathcal{H}_{+}^{m+1}(A), \quad b_{+s}^{m}(A) \stackrel{\text { def }}{=} \operatorname{dim} \mathcal{H}_{+s}^{m}(A), \\
& b_{+}^{m}(A) \stackrel{\text { def }}{=} b_{+s}^{m}(A) \text { when it is independent of } s, \\
& b_{+}^{m-1}(A) \stackrel{\text { def }}{=} \operatorname{dim} \mathcal{H}_{+}^{m-1}(A), \quad b^{j}(A) \stackrel{\operatorname{def}}{=} \operatorname{dim} \mathcal{H}^{j}(A) .
\end{aligned}
$$

Recall that a complex connection $A$ on a complex bundle $E$ induces a covariant derivative $d_{A}$ on the conjugate bundle $\bar{E}$ defined by $d_{A} f=\overline{d_{A} \bar{f}}$, where $f$ is a section of $\bar{E}$. Since $*_{l} \alpha=-i \alpha$ is equivalent to $*_{l} \bar{\alpha}=i \bar{\alpha}$, we have $\overline{\Omega_{+}^{m}(E)}=\Omega_{-}^{m}(E)$, $d_{A}^{++s} \omega=\overline{d_{A}^{--\bar{s}} \bar{\omega}}$ and $d_{A}^{+s} \omega=\overline{d_{A}^{-\bar{s}} \bar{\omega}}$. Thus we get 
Proposition 4.5. For an oriented closed smooth $(2 m+1)$-manifold $M$ with odd $m$, we have

$$
\begin{gathered}
\mathcal{H}^{j}(A)=\overline{\mathcal{H} j}(A), \quad \mathcal{H}_{-}^{m-1}(A)=\overline{\mathcal{H}_{+}^{m-1}(A)}, \\
\mathcal{H}_{-\bar{s}}^{m}(A)=\overline{\mathcal{H}_{+s}^{m}(A)}, \quad \mathcal{H}_{-}^{m+1}(A)=\overline{\mathcal{H}_{+}^{m+1}(A)}, \\
b^{j}(A)=b^{j}(A), \quad b_{-}^{m-1}(A)=b_{+}^{m-1}(A), \\
b_{-\bar{s}}^{m}(A)=b_{+s}^{m}(A), \quad b_{-}^{m+1}(A)=b_{+}^{m+1}(A), \\
\mathcal{H}_{-}^{m-1}(M)=\overline{\mathcal{H}_{+}^{m-1}(M),} \quad \mathcal{H}_{-\bar{s}}^{m}(M)=\overline{\mathcal{H}_{+s}^{m}(M)}, \quad \mathcal{H}_{-}^{m+1}(M)=\overline{\mathcal{H}_{+}^{m+1}(M),} \\
b_{-}^{m-1}(M)=b_{+}^{m-1}(M), \quad b_{-\bar{s}}^{m}(M)=b_{+s}^{m}(M), \quad b_{-}^{m+1}(M)=b_{+}^{m+1}(M),
\end{gathered}
$$

where $0 \leq j \leq m-2$. The above identities still hold if the term $\mathcal{H}$ is replaced by $H$ for self-duality cohomology groups with odd $m$ in Definition 4.1.

By the standard theory of elliptic operators on oriented Riemannian closed manifolds, we get the following Hodge decompositions.

Theorem 4.6. Let $M$ be an oriented closed manifold with the data $\left(H, o_{l}, o, g\right)$ as in Convention 1.1, and let $A$ be a connection on a real or complex vector bundle $E$ over $M$ with a Riemannian or Hermitian metric. If the complex in Theorems 1.3, 1.6, 2.2 and 2.4 is well-defined, then we have

$$
\mathcal{H}_{+}^{m-1}(A) \cong H_{+}^{m-1}(A), \quad \mathcal{H}_{+s}^{m}(A) \cong H_{+s}^{m}(A), \quad \mathcal{H}_{+}^{m+1}(A) \cong H_{+}^{m+1}(A),
$$

which all are finite dimensional, and further

$$
\begin{gathered}
\Omega^{m-1}(E)=\mathcal{H}_{+}^{m-1}(A) \oplus d_{A}\left(\Omega^{m-2}(E)\right) \oplus d_{A}^{*}\left(\Omega_{++}^{m}(E)\right), \\
\Omega_{++}^{m}(E)=\mathcal{H}_{+s}^{m}(A) \oplus d_{A}^{++s}\left(\Omega^{m-1}(E)\right) \oplus\left(d_{A}^{+s}\right)^{*}\left(\Omega_{+}^{m+1}(E)\right), \\
\Omega_{+}^{m+1}(E)=\mathcal{H}_{+}^{m+1}(A) \oplus d_{A}^{+}\left(\Omega_{++}^{m}(E)\right) .
\end{gathered}
$$

As usual for $0 \leq j \leq m-2, \mathcal{H}^{j}(A) \cong H^{j}(A)$, and

$$
\Omega^{j}(E)=\mathcal{H}^{j}(A) \oplus d_{A}\left(\Omega^{j-1}(E)\right) \oplus d_{A}^{*}\left(\Omega^{j+1}(E)\right) .
$$

Under the conditions of this theorem, $\mathcal{H}_{+s}^{m}(A) \cong H_{+s}^{m}(A)$ are isomorphic to each other for all nonzero $s$. Thus $b_{+s}^{m}(A) \stackrel{\operatorname{def}}{=} \operatorname{dim}_{+}^{m}(A)=\operatorname{dim} H_{+s}^{m}(A)$ is independent of $s$, according to Remark 4.2. This fact is not obvious from the definition of $\mathcal{H}_{+s}^{m}(A)$.

By using the Atiyah-Singer index theorem on closed odd-dimensional manifolds ([4], Proposition 9.2.), we get the following universal identities.

Theorem 4.7. Suppose the conditions of Theorem 4.6 hold. Then

$$
\begin{aligned}
b_{+}^{m-1}(M)-b_{+}^{m}(M)+b_{+}^{m+1}(M) & =(-1)^{m} \sum_{j=0}^{m-2}(-1)^{j} b^{j}(M), \\
b_{+}^{m-1}(A)-b_{+}^{m}(A)+b_{+}^{m+1}(A) & =(-1)^{m} \sum_{j=0}^{m-2}(-1)^{j} b^{j}(A) .
\end{aligned}
$$

The significance of these identities is that the left sides a priori depend on the data $\left(H, o_{l}, o, g\right)$, while the right sides are independent of these data. 


\section{ON COMPACT ODD-DIMENSIONAL MANIFOLDS WITH BOUNDARY}

In this section, let $M$ be a smooth compact $(2 m+1)$-manifold with boundary and with the data $\left(H, o_{l}, g_{l}, L\right)$ as in Convention 1.1. For the sake of brevity of statements, we denote complexes in Theorem 1.3, 1.6, 2.2 and 2.4 as:

$$
0 \rightarrow U^{0} \stackrel{D_{0}}{\rightarrow} \cdots \rightarrow U^{m-1} \stackrel{D_{m-1}}{\rightarrow} U^{m} \stackrel{D_{m}}{\rightarrow} U^{m+1} \rightarrow 0 .
$$

Explicitly, $D_{j}=d_{A}$ for $0 \leq j \leq m-2, D_{m-1}=d_{A}^{++s}=l_{s} d_{A}^{++}, D_{m}=d_{A}^{+s}=d_{A}^{+} h_{s}$ and $D_{m+1}=D_{-1}=0$.

Let $E$ be equipped with a $d_{A}$-parallel-invariant metric. By (4.4), the formal adjoint operator $D_{j}^{*}$ of $D_{j}$ is a local operator. Explicitly, $D_{j}^{*}=d_{A}^{*}$ for $0 \leq j \leq m-2$, $D_{m-1}^{*}=\left(d_{A}^{++s}\right)^{*}=d_{A}^{*} l_{\bar{s}}, D_{m}^{*}=\left(d_{A}^{+s}\right)^{*}=h_{\bar{s}} \pi^{++} d_{A}^{*}$ and $D_{m+1}^{*}=D_{-1}^{*}=0$.

Recall that $\omega_{h} \stackrel{\text { def }}{=} \pi_{h} \omega$ and $\omega_{l} \stackrel{\text { def }}{=} \pi_{l} \omega$ denote the flow component and level component of a form $\omega$ respectively. Let $K$ be the union of some components of boundary $\partial M . K$ may be empty. The b-boundary condition on $\omega$ in $U^{j}$ is defined as:

$$
\omega_{l}=0 \text { on } K, \quad \omega_{h}=0 \text { on } \partial M-K .
$$

Definition 5.1. Let $M$ be a compact oriented smooth $(2 m+1)$-manifold with boundary and with the data $\left(H, o_{l}, o, g\right)$ as in Convention 1.1. Let $0 \leq j \leq m+1$. Define

$$
\mathcal{H}_{b}^{j} \stackrel{\text { def }}{=} \operatorname{Ker} D_{j} \cap \operatorname{Ker} D_{j-1}^{*} \text { in } U_{b}^{j}
$$

Explicitly, it is written as $\mathcal{H}_{+}^{m-1}(A, K)$ for $j=m-1, \mathcal{H}_{+s}^{m}(A, K)$ for $j=m$, $\mathcal{H}_{+}^{m+1}(A, K)$ for $j=m+1$, and $\mathcal{H}^{j}(A, K)$ for $0 \leq j \leq m-2$. Moreover we define

$$
\begin{aligned}
& b_{+}^{m+1}(A, K) \stackrel{\text { def }}{=} \operatorname{dim} \mathcal{H}_{+}^{m+1}(A, K), \quad b_{+s}^{m}(A, K) \stackrel{\text { def }}{=} \operatorname{dim} \mathcal{H}_{+s}^{m}(A, K), \\
& b_{+}^{m}(A, K) \stackrel{\text { def }}{=} b_{+s}^{m}(A, K) \text { when it is independent of } s, \\
& b_{+}^{m-1}(A, K) \stackrel{\text { def }}{=} \operatorname{dim} \mathcal{H}_{+}^{m-1}(A, K), \quad b^{j}(A, K) \stackrel{\text { def }}{=} \operatorname{dim} \mathcal{H}^{j}(A, K) .
\end{aligned}
$$

Remark 5.2. When $d_{A}=d$, we replace the term $A$ by $M$ in the above notations. When $K$ is empty, we omit the term $K$ in the above notations, and thus get notations such as $\mathcal{H}_{+}^{m}(A)$. This $\mathcal{H}_{+}^{m}(A)$ for a compact manifold with boundary should not be confused with the $\mathcal{H}_{+}^{m}(A)$ in Definition 4.4, which is for a closed manifold.

The $\bar{b}$-boundary condition on $\omega$ in $U^{j}$ is defined as:

$$
\omega_{l}=0 \text { and }\left(D_{j-1}^{*} \omega\right)_{l}=0 \text { on } K, \quad \omega_{h}=0 \text { and }\left(D_{j} \omega\right)_{h}=0 \text { on } \partial M-K .
$$

Let $U_{b}^{j}, U_{\dot{b}}^{j}, U_{\bar{b}}^{j}$ denote the space of forms in $U^{j}$ with the $b, \dot{b}, \bar{b}$-boundary conditions respectively. The $\dot{b}$-boundary condition will be defined soon.

The Laplacian operator on the $j$-th stage of complex (5.1) is denoted by $\triangle_{j}=$ $D_{j-1} D_{j-1}^{*}+D_{j}^{*} D_{j}$. Explicitly these Laplacian operators are written as:

$$
\begin{aligned}
& \triangle_{m+1, A}^{+s}=d_{A}^{+s}\left(d_{A}^{+s}\right)^{*} \\
& \triangle_{m, A}^{+s}=d_{A}^{++s}\left(d_{A}^{++s}\right)^{*}+\left(d_{A}^{+s}\right)^{*} d_{A}^{+s}, \\
& \triangle_{m-1, A}^{+s}=d_{A} d_{A}^{*}+\left(d_{A}^{++s}\right)^{*} d_{A}^{++s}, \\
& \triangle_{j, A}=d_{A} d_{A}^{*}+d_{A}^{*} d_{A} .
\end{aligned}
$$


Now we state a main theorem in this paper.

Theorem 5.3. Let $M$ be a compact oriented smooth $(2 m+1)$-manifold with boundary and with the data $\left(H, o_{l}, o, g\right)$ as in Convention 1.1 such that $H$ is transverse to $\partial M$, and let $A$ be a connection on a real or complex vector bundle $E$ with a $d_{A}$-parallel-invariant metric. Then the system $\triangle_{j} \omega=0$ on $M$ with the $\bar{b}$-boundary condition on $\omega$ is an elliptic boundary system. In particular, Ker $\triangle_{j}$ in $U_{\bar{b}}^{j}$ is finite dimensional.

The proof of this theorem is postponed to Section 9. Plainly, if $D_{j} \omega=0$ and $D_{j-1}^{*} \omega=0$ then $\triangle_{j} \omega=0$. For the converse, we have

Lemma 5.4. Assume $H \perp_{g} \partial M$. If $\omega_{l}=0$ on $K, \omega_{h}=0$ on $\partial M-K,\left(D_{j-1}^{*} \eta\right)_{l}=$ 0 on $K$ and $\left(D_{j} \eta\right)_{h}=0$ on $\partial M-K$, then $\left(D_{j} \omega, D_{j} \eta\right)+\left(D_{j-1}^{*} \omega, D_{j-1}^{*} \eta\right)=\left(\omega, \triangle_{j} \eta\right)$. In particular,

(1) if both $\omega$ and $\eta$ satisfy the $\bar{b}$-boundary condition, then $\left(\omega, \triangle_{j} \eta\right)=\left(\triangle_{j} \omega, \eta\right)$;

(2) $\triangle_{j} \omega=0$ on $M$ and $\omega \in U_{\bar{b}}^{j}$ if and only if $D_{j} \omega=0$ and $D_{j-1}^{*} \omega=0$ on $M$ and $\omega \in U_{b}^{j}$.

This lemma can be easily proved by Green's formula

$$
\left(D_{j} \omega, \eta\right)-\left(\omega, D_{j}^{*} \eta\right)=\int_{\partial M} \omega_{l} \wedge * \eta_{h},
$$

for $\omega \in U_{j}, \eta \in U_{j}$ and $-1 \leq j \leq m+1$. For this we just use the standard Green's formula: $\left(d_{A} \omega, \eta\right)-\left(\omega, d_{A}^{*} \eta\right)=\int_{\partial M} i^{*}(\omega \wedge * \eta)=\int_{\partial M} \omega_{l} \wedge * \eta_{h}$, where $i$ denotes the inclusion map of $\partial M$ into $M$ and $H \perp_{g} \partial M$. Note $* \eta_{h}$ is always a level form according to Lemma 4.3, and thus is a tangential form, since $H \perp_{g} \partial M$.

In order to define finite dimensional self-dual cohomology groups of a half De Rham complex on a compact manifold with boundary, we need a suitable boundary condition. The $\dot{b}$-boundary condition on $\omega$ in $U^{j}$ is defined as:

$$
\omega_{l}=0 \text { on } K, \quad \omega_{h}=0 \text { and }\left(D_{j} \omega\right)_{h}=0 \text { on } \partial M-K .
$$

Lemma 5.5. Suppose $L$ is tangent to $\partial M$, i.e., $H \perp_{g} \partial M$. Let $\eta \in U^{j}$, and let $W$ be an open subset $W$ of $\partial M$.

(1) If $\eta_{l}=0$ on $W$ then $\left(D_{j} \eta\right)_{l}=0$ on $W$.

(2) If $\eta_{h}=0$ on $W$ then $\left(D_{j}^{*} \eta\right)_{h}=0$ on $W$.

The above lemma follows easily from the following formulas.

Lemma 5.6. Let $(M, H, L),(U, \tau, X)$ and $(\omega, \alpha, \beta)$ be the same as in Lemma 3.3. Suppose $N$ is a one-to-one immersed codimension-one submanifold in $U$ and $N$ is tangent to the $L$. Then

(1) $d_{l} \tau=0$ as a section of $\left.\wedge^{2} L^{*}\right|_{N}$ over $N$.

(2) $d_{A}(\tau \wedge \beta+\alpha)=\tau \wedge\left[\nabla_{A_{X}} \alpha-d_{A_{l}} \beta+\left(\nabla_{X} \tau\right) \beta\right]+d_{A_{l}} \alpha$ as sections of $\left.\wedge^{j} T^{*} M\right|_{N} \otimes$ $\left.E\right|_{N}$ over $N$.

(3) $d_{A_{l}} \omega=d_{A_{l}} \pi_{l} \omega$ and $\pi_{l} d_{A}^{k} \omega=\left(d_{A_{l}}\right)^{k} \pi_{l} \omega$ as sections of $\left.\left.\wedge^{j} T^{*} M\right|_{N} \otimes E\right|_{N}$ over $N$, for all $k \geq 0$.

Proof. Let $k$ denote the inclusion map from $N$ into $U$. Since $N$ is tangent to $L$, we have

$$
k_{*} T N=\left.L\right|_{N}
$$


Thus $\left\langle k^{*} \tau, T N\right\rangle=\left\langle\tau, k_{*} T N\right\rangle=\left\langle\tau,\left.L\right|_{N}\right\rangle=0$, and $k^{*} \tau$ is a zero form on $N$, so $d_{N} k^{*} \tau=0$ over $N$, where $d_{N}$ denotes the exterior derivative on $N$. Note that $\left\langle d_{h} \tau, \wedge^{2} L\right\rangle=0$ on $U$, since $d_{h} \tau \in H^{*} \wedge L$. Thus over $N$ we have

$$
\left\langle d_{l} \tau,\left.\wedge^{2} L\right|_{N}\right\rangle=\left\langle d \tau, k_{*} \wedge^{2} T N\right\rangle=\left\langle k^{*} d \tau, \wedge^{2} T N\right\rangle=\left\langle d_{N} k^{*} \tau, \wedge^{2} T N\right\rangle=0 .
$$

Since $\left\langle d_{l} \tau,\left.(H \wedge L)\right|_{N}\right\rangle=0$ always holds, we have $d_{l} \tau=0$ as a section of $\left.\wedge^{2} L^{*}\right|_{N}$. The remaining formulas of Lemma 5.6 follow rather easily from the fact $d_{l} \tau=0$ over $N$ and Lemma 3.3.

According to Lemma 5.5, the $\dot{b}$-boundary condition is well-defined on the complex (5.1), in the case when $L$ is tangent to $\partial M$, i.e., $H \perp_{g} \partial M$. Now we make

Definition 5.7. Let $M$ be a compact smooth $(2 m+1)$-manifold with boundary and with the data $\left(H, o_{l}, c_{l}, L\right)$ as in Convention 1.1 such that the $L$ is tangent to $\partial M$. When the complex in Theorems 1.3, 1.6, 2.2 and 2.4 is well-defined, the cohomology groups of the following complex (5.8) are denoted by $H^{j}(A, K), H_{+}^{m-1}(A, K)$, $H_{+s}^{m}(A, K)$ and $H_{+}^{m+1}(A, K)$, where $0 \leq j \leq m-2$. They are called (relative) self-duality cohomology groups.

$$
0 \rightarrow U_{\dot{b}}^{0} \stackrel{D_{0}}{\rightarrow} \cdots \stackrel{D_{m-2}}{\rightarrow} U_{\dot{b}}^{m-1} \stackrel{D_{m-1}}{\rightarrow} U_{\dot{b}}^{m} \stackrel{D_{m}}{\rightarrow} U_{\dot{b}}^{m+1} \rightarrow 0 .
$$

Note that $h_{s}\left(H_{+s}^{m}(A, K)\right)=H_{+1}^{m}(A, K)$, as in Remark 4.2. Remark 5.2 applies similarly to the above notations. For the sake of brevity, we let

$$
H_{b}^{j} \stackrel{\text { def }}{=} \frac{\operatorname{Ker} D_{j} \text { in } U_{\dot{b}}^{j}}{D_{j-1}\left(U_{\dot{b}}^{j-1}\right)} .
$$

Now we can state the following Hodge decomposition theorem, using the notations defined in this section.

Theorem 5.8. Let $\left(M, H, o_{l}, o, g\right)$ and $(E, A)$ be the same as in Theorem 5.3. If $H \perp_{g} \partial M$ and the complex in Theorems 1.3, 1.6, 2.2 and 2.4 is well-defined, then for all $0 \leq j \leq m+1$, we have

$$
\begin{gathered}
\mathcal{H}_{b}^{j} \cong H_{b}^{j}, \\
U^{j}=\mathcal{H}_{b}^{j} \oplus D_{j-1} U_{b}^{j-1} \oplus D_{j}^{*} U_{b}^{j+1}, \\
U_{b}^{j}=\mathcal{H}_{b}^{j} \oplus D_{j-1} U_{\bar{b}}^{j-1} \oplus D_{j}^{*} U_{\bar{b}}^{j+1} .
\end{gathered}
$$

The proof of this theorem is given in Section 9. Under the conditions of this theorem, $\mathcal{H}_{+s}^{m}(A, K) \cong H_{+s}^{m}(A, K)$ are isomorphic to each other for all nonzero $s$, and $b_{+s}^{m}(A, K)$ is independent of $s$. By the same reasoning as in Proposition 4.5, we get 
Proposition 5.9. For an oriented compact smooth $(2 m+1)$-manifold with boundary and with odd $m$, we have

$$
\begin{array}{ll}
\mathcal{H}^{j}(A, K)=\overline{\mathcal{H}^{j}(A, K)}, & \mathcal{H}_{-}^{m-1}(A, K)=\overline{\mathcal{H}_{+}^{m-1}(A, K)}, \\
\mathcal{H}_{-\bar{s}}^{m}(A, K)=\overline{\mathcal{H}_{+s}^{m}(A, K),} & \mathcal{H}_{-}^{m+1}(A, K)=\overline{\mathcal{H}_{+}^{m+1}(A, K)}, \\
b^{j}(A, K)=b^{j}(A, K), & b_{-}^{m-1}(A, K)=b_{+}^{m-1}(A, K), \\
b_{-\bar{s}}^{m}(A, K)=b_{+s}^{m}(A, K), & b_{-}^{m+1}(A, K)=b_{+}^{m+1}(A, K), \\
\mathcal{H}_{-}^{m-1}(M, K)=\overline{\mathcal{H}_{+}^{m-1}(M, K),} & \mathcal{H}_{-\bar{s}}^{m}(M, K)=\overline{\mathcal{H}_{+s}^{m}(M, K),} \\
\mathcal{H}_{-}^{m+1}(M, K)=\overline{\mathcal{H}_{+}^{m+1}(M, K),} & b_{-}^{m-1}(M, K)=b_{+}^{m-1}(M, K), \\
b_{-\bar{s}}^{m}(M, K)=b_{+s}^{m}(M, K), & b_{-}^{m+1}(M, K)=b_{+}^{m+1}(M, K),
\end{array}
$$

where $0 \leq j \leq m-2$. The above identities still hold if the term $\mathcal{H}$ is replaced by $H$ for self-duality cohomology groups for odd $m$ in Definition 5.7.

\section{Conformal Line Fields ANd SelF-DUAl Line FieldS}

In this section we study the assumptions on the $T_{X}$ in Theorems 1.3, 1.6, 2.2, 2.4. Since in defining $T_{X}$ we only use the data $\left(H, o_{l}, g_{l}\right)$, the properties of $T_{X}$ depend only on $\left(H, o_{l}, g_{l}\right)$, and have nothing to do with the data $L$.

It is not neccessary to assume $\operatorname{dim} H=1$ for the disussion in this section. Let $H$ be a smooth tangent $(n-q)$-plane field over a smooth $n$-manifold $M$. It induces a dual cotangent $q$-plane field $L^{*} \stackrel{\text { def }}{=} H^{\perp}$, and also induces an intrinsic quotient bundle $Q \stackrel{\text { def }}{=} T M / H$. Let $[Y]$ denote the equivalence class of $Y$ in $T M / H$ for $Y \in T M$. Since the pairing $\langle\alpha,[Y]\rangle \stackrel{\text { def }}{=}\langle\alpha, Y\rangle$ is well-defined for any $\alpha \in L^{*}$, we have a canonical identification

$$
L^{*}=Q^{*} \stackrel{\text { def }}{=} \operatorname{Hom}_{\mathbb{R}}(T M / H, \mathbb{R}) .
$$

Suppose $H$ is integrable. This is always true if $\operatorname{dim} H=1$. Let $X$ be a section of $H$ over $M$. Then $X$ is a vector field and generates a 1-parameter group of local diffeomorphisms $\phi_{t}$ of $M$. Since $H$ is integrable, $\phi_{t *}$ preserves $H$ and, dually, $\phi_{t}^{*}$ preserves $L^{*}$. Thus $L_{X} \alpha$ is a section of $L^{*}$ if $\alpha$ is a section of $L^{*}$. The holonomytranslation induced by $H$ is the action on elements in $L^{*}$ by the differentials of $\phi_{t}$. There is also another equivalent way to define holonomy-translations. (For example, see [5], pp. 81-82; [11], pp. 24-25.)

A smooth foliation is called Riemannian if there is a smooth holonomy invariant Riemannian metric $g_{l}$ on the quotient bundle. Here 'holonomy invariant' means $g_{l}$ is invariant under the holonomy-translation along paths in the leaves of the foliation, i.e., $L_{X} g_{l}=0$ for any vector field $X$ tangent to the foliation. An integrable smooth plane field $H$ is called Riemannian if its integral foliation is Riemannian.

Proposition 6.1. Suppose an integrable smooth plane field $H$ is Riemannian with a smooth holonomy invariant metric $g_{l}$ on the dual $L^{*} \stackrel{\text { def }}{=} H^{\perp}$. Then

(1) $g_{l}\left(\phi_{t}^{*} \alpha, \phi_{t}^{*} \beta\right)=g_{l}(\alpha, \beta)$ for any vector field $X$ in $H$ and any $\alpha$ and $\beta$ in the fiber $L_{x}^{*}$ at a point $x$ of $M$.

(2) If $\rho^{1}, \ldots, \rho^{j}$ is an orthonormal frame of $\left(L^{*}, g_{l}\right)$ at $x$, then $\phi_{t}^{*}\left(\rho^{1}\right), \ldots, \phi_{t}^{*}\left(\rho^{j}\right)$ is an orthonormal frame of $\left(L, g_{l}\right)$ at $\phi_{t}(x)$. In particular the level-star torsion 
operator

$$
T_{X} \stackrel{\text { def }}{=} L_{X} *_{l}-*_{l} L_{X}=0
$$

on all smooth sections of $\wedge^{j} L^{*}$ for all $j$.

Proof. The above statement (1) just means $g_{l}$ is holonomy invariant. We need only to check the statement (2). If $\left\{\rho^{1}, \ldots, \rho^{j}\right\}$ is an orthonormal frame of $\left(L, g_{l}\right)$ at point $x$, then $\left\{\phi_{t}^{*}\left(\rho^{1}\right), \ldots, \phi_{t}^{*}\left(\rho^{j}\right)\right\}$ is an orthonormal fame of $\left(L, g_{l}\right)$ at the point $\phi_{t}(x)$. Let $\rho_{t}^{I}$ denote $\phi_{t}^{*}\left(\rho^{i_{1}}\right) \wedge \ldots \wedge \phi_{t}^{*}\left(\rho^{i_{j}}\right)$ with $1 \leq j \leq p$. Then $*_{l} \rho_{t}^{I}=\rho_{t}^{I^{\prime}}$, where $I I^{\prime}$ is an even permutation. Thus $L_{X} *_{l} \rho_{t}^{I}=L_{X} \rho_{t}^{I^{\prime}}=0=*_{l} 0=*_{l} L_{X} \rho_{t}^{I}$. Therefore $L_{X} *_{l}=*_{l} L_{X}$ on all smooth sections of $\wedge^{j} L^{*}$ for all $j$.

A smooth foliation is called conformal if it can be described by a family of distinguished charts $\left\{U_{\lambda}, f_{\lambda}, \gamma_{\lambda \nu}^{x}\right\}$, where $f_{\lambda}$ is a smooth submersion of $U_{\lambda}$ to $\mathbb{R}^{q}$ with some Riemannian metric $g_{\lambda}$, and $\gamma_{\lambda \nu}^{x}$ is a local conformal mapping from a neighborhood of $f_{\lambda}(x)$ onto a neighborhood of $f_{\nu}(x)$ such that $f_{\lambda}=\gamma_{\lambda \nu}^{x} f_{\nu}$ on some neighborhood of $x$ in $U_{\lambda} \cap U_{\nu}$. An integrable smooth plane field $H$ is called conformal if its integral foliation is so. Proposition 1.1 of [14] states that an integrable smooth tangent plane field $H$ of codimension $q$ is conformal if and only if there is a smooth positive definite level-metric $g_{l}$ on $L^{*} \stackrel{\text { def }}{=} H^{\perp}$ such that any point in $M$ has a neighborhood $U$ on which $\left.g_{l}\right|_{U}$ is locally conformal to a holonomy invariant metric on $\left.L^{*}\right|_{U}$. The proof in fact shows there are sufficiently many such level-metrics. But for a clear statement, we make the following definition first.

Definition 6.2. A local conformal data $\left\{U_{\lambda}, \bar{U}_{\lambda}, g_{\lambda}, f_{\lambda}, \phi_{\lambda \nu}, \rho_{\lambda}, \psi_{\lambda}\right\}$ of an integrable plane field $H$ of codimension $q$ means the following:

$\left\{U_{\lambda}\right\}$ is an open cover of $M ; \bar{U}_{\lambda}$ is an smooth $q$-manifold which is diffeomorphic to $\mathbb{R}^{q} ; g_{\lambda}$ is a smooth Riemannian metric on $\bar{U}_{\lambda} ; f_{\lambda}$ is a smooth submersion from $U_{\lambda}$ to $\bar{U}_{\lambda} ; \phi_{\lambda \nu}$ is a smooth function on $U_{\lambda} \cap U_{\nu}$ for any pair $(\lambda, \nu)$ such that $U_{\lambda} \cap U_{\nu}$ is nonempty, and all these $\left\{\phi_{\lambda \nu}\right\}$ together satisfy

$$
f_{\lambda}^{*} g_{\lambda}=e^{\phi_{\lambda \nu}} f_{\nu}^{*} g_{\nu} \quad \text { on } U_{\lambda} \cap U_{\nu}
$$

$\rho_{\lambda}: M \rightarrow R$ is a smooth function supported in $U_{\lambda}$ such that

$$
\sum_{\text {all } \lambda} \rho_{\lambda}(x)=1 \quad \text { for any } x \in M \text {; }
$$

and $\psi_{\lambda}: U_{\lambda} \rightarrow R$ is a smooth function defined as $\psi_{\lambda}(x) \stackrel{\text { def }}{=} \sum_{w} \rho_{w}(x) \phi_{w \lambda}(x)$ for $x \in U_{\lambda}$.

Note the above $\psi_{\lambda}$ is well-defined, since if $x \in U_{\lambda}$ and $x \notin U_{w}$ then $\rho_{w}(x)=0$, and if $x \in U_{\lambda} \cap U_{w}$ then $\rho_{w}(x) \phi_{w \lambda}(x)$ is already well-defined. Also note that $\rho_{\lambda}$ is not assumed to be positive.

Remark 6.3. An integrable plane field $H$ is conformal if and only if the local conformal data exist.

(1) We have $\phi_{\lambda \nu}(x)=\psi_{\nu}(x)-\psi_{\lambda}(x)$ for $x \in U_{\lambda} \cap U_{\nu}$, since $\phi_{\lambda \nu}(x)+\phi_{\nu w}(x)=$ $\phi_{\lambda w}(x)$ for $x \in U_{\lambda} \cap U_{\nu} \cap U_{w}$.

(2) Given the cover $\left\{U_{\lambda}\right\}$, define $I\left(U_{\lambda}\right) \stackrel{\text { def }}{=} U_{\lambda}-\cup_{\nu \neq \lambda} U_{\mu}$, which is the part of $U_{\lambda}$ not intersecting any other $U_{\nu}$ 's. If $x \in I\left(U_{\lambda}\right)$, then $\rho_{\lambda}(x)=1$ and $\psi_{\lambda}(x)=1$.

(3) Given any $x \in M$, we can choose $\left\{U_{\lambda}\right\}$ such that all nonempty overlaps $U_{\lambda} \cap U_{\nu}$ are sufficiently small so that there is an open neighbourhood $W_{x}$ of $x$ with 
closure $\bar{W}_{x} \subset I\left(U_{\lambda}\right)$ for some $\lambda$. Then the $\left.g_{\lambda}\right|_{W_{x}}$ can be arbitrarily chosen without any influenece on the equation (6.2), because it is only required on the nonempty overlaps. Since $\psi_{\lambda}=1$ on $W_{x} \subset I\left(U_{\lambda}\right)$, we can make the metric $e^{\psi_{\lambda}} f_{\lambda}^{*} g^{\lambda}=f_{\lambda}^{*} g^{\lambda}$ on $\left.L^{*}\right|_{W_{x}}$ over $W_{x}$ to be arbitrarily large or small, and is holonomy invariant too. In addition, we can obviously arrange $W_{x}$ to be a simple open subset. Therefore we obtain the following Proposition 6.4. (Recall that calling $U$ a simple open set means that the foliation restricted to $U$ is induced by a smooth submersion $\pi$ of $U$ to some smooth manifold $\bar{U}$ such that any fiber of $\pi$ is connected. See [11], pp. 14-15.)

Proposition 6.4. Suppose an integrable smooth plane field $H$ is conformal. Then for any local conformal data $\left\{U_{\lambda}, \bar{U}_{\lambda}, g_{\lambda}, f_{\lambda}, \phi_{\lambda \nu}, \rho_{\lambda}, \psi_{\lambda}\right\}$, the level-metric $g_{\lambda}$ defined as

$$
g_{l} \stackrel{\text { def }}{=} e^{\psi_{\lambda}} f_{\lambda}^{*} g^{\lambda} \quad \text { on } U_{\lambda}
$$

is a global smooth positive definite level-metric on $L^{*}$ over the whole manifold $M$. Furthermore for any given $x \in M$, we can choose a local conformal data and choose a simple open neighbourhood $W_{x}$ of $x$ so that the $\left.g_{l}\right|_{W_{x}}$ is arbitrarily large or small and is holonomy invariant as well. If in addition codim $H=2 m$, then all the level-metrics $g_{l}$ on $L^{*}$ over $M$ defined by (6.4) are compatible, namely, $T_{X}=L_{X} *_{l}-*_{l} L_{X}=0$ on the level middle-dimensional $\wedge^{m} L^{*}$.

Now we want to define a notion which, in general, is weaker than the notion of a conformal foliation but suffices to guaranttee the condition of $T_{X}$ in Theorems 1.3, 1.6, 2.2 and 2.4. First we have the following lemma.

Lemma 6.5. Let $H$ be an integrable smooth tangent plane field of codimension $2 m$. Let $L^{*} \stackrel{\text { def }}{=} H^{\perp}$ be its dual cotangent plane field with an orientation $o_{l}$. Then the following three statements are equivalent.

(1) $T_{X} \stackrel{\text { def }}{=} L_{X} *_{l}-*_{l} L_{X}=0$ on $\wedge^{m} L^{*}$.

(2) $T_{X}=0$ on $\wedge_{-}^{m} L^{*}$ for even $m$ or $\wedge_{-}^{m} L_{c}^{*}$ for odd $m$.

(3) $T_{X}=0$ on $\wedge_{+}^{m} L^{*}$ for even $m$ or $\wedge_{+}^{m} L_{c}^{*}$ for odd $m$.

Proof. Obviously, we need only to prove that the statement (2) is equivalent to the

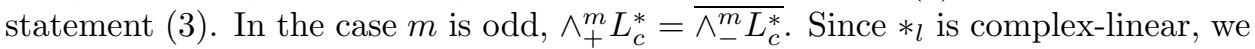
have $\overline{{ }_{l} \omega}=*_{l} \bar{\omega}$. Thus $T_{X} \omega=0$ if and only if $T_{X} \bar{\omega}=\overline{T_{X} \omega}=0$.

In the case $m$ is even, let $\wedge_{-}, \wedge_{+}$denote the space of sections of $\wedge_{-}^{m} L^{*}$ and $\wedge_{+}^{m} L^{*}$ repectively. Then for all $\omega \in \wedge_{-}$and $\eta \in \wedge_{+}$, we have $\omega \wedge \eta=\omega \wedge *_{l} \eta=$ $\langle\omega, \eta\rangle \cdot \operatorname{vol}\left(g_{l}\right)=0$, and $\left(L_{X} \omega\right) \wedge \eta=-\omega \wedge L_{X} \eta$. Therefore the statement that $L_{X} \omega \in \wedge_{-}$whenever $\omega \in \wedge_{-}$is equivalent to the statement that $L_{X} \eta \in \wedge_{+}$ whenever $\eta \in \wedge_{+}$.

Note that, for any $\omega \in \wedge_{-}, T_{X} \omega=L_{X} *_{l} \omega-*_{l} L_{X} \omega=-L_{X} \omega-*_{l} L_{X} \omega$. Thus $T_{X}=0$ on $\wedge_{-}$just means that $L_{X} \omega \in \wedge_{-}$whenever $\omega \in \wedge_{-}$. Similarly, $T_{X}=0$ on $\wedge_{+}$just means that $L_{X} \omega \in \wedge_{+}$whenever $\omega \in \wedge_{+}$. Therefore we have proved the statement (2) is equivalent to the statement (3).

Definition 6.6. An integrable tangent plane field $H$ of codimension $2 m$ is called self-dual if there is a smooth metric $g_{l}$ and an orientation $o_{l}$ on the dual $L^{*} \stackrel{\text { def }}{=} H^{\perp}$ such that $T_{X} \stackrel{\text { def }}{=} L_{X} *_{l}-*_{l} L_{X}=0$ on $\wedge^{m} L^{*}$. Such a level metric $g_{l}$ and its conformal class $\left[g_{l}\right]$ are called compatible. 
Corollary 6.7. In Theorems 1.3, 1.6, 2.2 and 2.4, if the $c_{l}$ is compatible, then the conditions on $T_{X}$ are satisfied for all connections $A$.

Remark 6.8. Proposition 6.4 tells us that if a smooth line field $H$ is conformal then it is self-dual. When the codimension of $H$ is 2 or 4 , the converse is also true. Indeed, we can choose $\bar{U}_{\lambda}$ to be sufficiently small local transversals of $H$ which are diffeomorphic to $\mathbb{R}^{2 m}$. These $\bar{U}_{\lambda}$ 's get the induced Riemannian metric from a compatible metric $g_{l}$ by the identification $T^{*} \bar{U}_{\lambda}=\left.L^{*}\right|_{\bar{U}_{\lambda}}$. Let $U_{\lambda}$ be a union of small segments of leaves of $H$ which are transverse to $\bar{U}_{\lambda}$. We can choose sufficiently many $\bar{U}_{\lambda}$ 's so that all these $U_{\lambda}$ 's together cover $M$. The submersion $f_{\lambda}$ is defined by the holonomy translation of a point in $U_{\lambda}$ to a point in $\bar{U}_{\lambda}$ induced by $H$. Each $\gamma_{\lambda \nu}^{x}$ from $\bar{U}_{\nu}$ to $\bar{U}_{\lambda}$ is induced by the holonomy translation. Since $g_{l}$ is compatible, each $\gamma_{\lambda \nu}^{x}$ obtained in this way preserves the middle-dimensional star operator, and hence must be a conformal mapping, because $\operatorname{codim} H=2$ or 4 . This is obvious if $\operatorname{codim} H=2$; for the case $\operatorname{codim} H=4$, see ([6], p. 8).

\section{Computations of $(m-1)$-TH AND $(m+1)$-TH SELF-DUALITY COHOMOLOGY GROUPS}

In this section, we compute the $(m-1)$-th and $(m+1)$-th self-duality cohomology groups in a general case. First we need some simple lemmas. Let $N$ be a smooth $2 m$-manifold. When we say that a smooth map $k: N \rightarrow M$ is transverse to $H$ at $x \in N$, we mean $H_{y}+k_{*} T_{x} N=T_{y} M$, where $y=k(x)$ and $H_{y}$ denotes the fiber of $H$ at $y$.

Lemma 7.1. Let $H$ be a tangent line field on a smooth $(2 m+1)$-manifold $M$, and let $N$ be a smooth $2 m$-manifold with a smooth map $k: N \rightarrow M$. If $k$ is transverse to $H$ at $x \in N$, then

(1) $H_{y} \cap k_{*} T_{x} N=\{0\}$ and $k_{*}: T_{x} N \rightarrow T_{y} M$ is injective, where $y=k(x)$.

(2) $k^{*}: L_{y}^{*} \rightarrow T_{x}^{*} N$ is an linear isomorphism.

Proof. The statement (1) follows from a dimension counting. To prove the statement (2), we need only prove $k^{*}$ is injective. Suppose $\omega \in L_{y}^{*}$ and $k_{*} \omega=0$. Then $0=\left\langle k_{*} \omega, T_{x} N\right\rangle=\left\langle\omega, k_{*} T_{x} N\right\rangle$. By the definition $L^{*} \stackrel{\text { def }}{=} H^{\perp}$, we have $\left\langle\omega, H_{y}\right\rangle=0$. Hence $\left\langle\omega, T_{y} M\right\rangle=0$, and thus $\omega=0$.

Let $g_{l}$ and $o_{l}$ be an inner product and an orientation on $L_{y}^{*}$. Then there is a unique inner product $g_{N}$ and an orientation $o_{N}$ on $T_{x}^{*} N$ such that $k^{*}$ becomes an orientation-preserving isometry. Let $\operatorname{vol}\left(g_{N}\right)$ and $\operatorname{vol}\left(g_{l}\right)$ be the oriented volume forms of $\left(g_{N}, o_{N}\right)$ on $T_{x}^{*} N$ and $\left(g_{l}, o_{l}\right)$ on $L_{y}^{*}$ respectively. Let $*_{N}$ and $*_{l}$ be the star operators of $\left(g_{N}, o_{N}\right)$ on $\wedge^{j} T_{x}^{*} N$, and $\left(g_{l}, o_{l}\right)$ on $\wedge^{j} L_{y}^{*}$ respectively. Since $k^{*}$ is an orientation-preserving isometry, we have

Lemma 7.2. Let $(M, H, N, k)$ be the same as in Lemma 7.1. If $k$ is transverse to $H$ at $x \in N$, then $k^{*} \operatorname{vol}\left(g_{l}\right)=\operatorname{vol}\left(g_{N}\right)$ and $*_{N} k^{*} \alpha=k^{*} *_{l} \alpha$ for any $\alpha \in \wedge^{j} L_{y}^{*}$.

Theorem 7.3. Let $M$ be a connected, smooth $(2 m+1)$-manifold with the data $\left(H, o_{l}, c_{l}\right)$ as in Convention 1.1. Assume (1) A is a flat connection on a vector bundle $E$ with a $d_{A}$-parallel-invariant metric; and (2) there is a nonempty closed orientable smooth $2 m$-manifold $N$ which is smoothly immersed in $M$ and is transverse to $H$ at any point of $N$. Then $d_{A} \omega=0$ on $M$ if and only if $d_{A}^{++} \omega=0$ for some smooth tangent codimension-one plane field $L$ which is transverse to $H$. Here $\omega$ is a smooth E-valued $(m-1)$-form on $M$. 
Proof. Recall that $d_{A} \omega=d_{A_{h}} \omega+d_{A_{l}} \omega$ and $d_{A}^{++} \omega=d_{A_{h}} \omega+d_{A_{l}}^{+} \omega$. Thus we need only to prove that if $d_{A}^{++} \omega=0$ on $M$, then $d_{A_{l}} \omega=0$ on $M$. There are three ways to get $d_{A_{l}} \omega=0$ at a point $x$ in $M$. Let $k$ be the immersion from $N$ into $M$.

(1) The point $x \in k(N)$. This is argued as follows. In the case $m$ is even, since $A$ is flat and $d_{A_{h}} \omega=0$ on $M$ and $d_{A_{l}}^{+} \omega=0$ on $M$, we have

$$
\begin{aligned}
-\int_{N} k^{*}\left|d_{A_{l}}^{-} \omega\right|^{2} k^{*} \operatorname{vol}\left(g_{l}\right) & =\int_{N} k^{*}\left(\left|d_{A_{l}}^{+} \omega\right|^{2}-\left|d_{A_{l}}^{-} \omega\right|^{2}\right) k^{*} \operatorname{vol}\left(g_{l}\right) \\
& =\int_{N} k^{*}\left(d_{A_{l}} \omega \wedge d_{A_{l}} \omega\right) \\
& =\int_{N} k^{*}\left(d_{A} \omega \wedge d_{A} \omega\right)=\int_{N} k^{*} d\left(\omega \wedge d_{A} \omega\right) \\
& =\int_{N} d_{N} k^{*}\left(\omega \wedge d_{A} \omega\right)=0 .
\end{aligned}
$$

Here $d_{N}$ denotes the exterior derivative on $N$. By Lemma 7.2, $k^{*} \operatorname{vol}\left(g_{l}\right)=\operatorname{vol}\left(g_{N}\right)$. Thus $k^{*}\left|d_{A_{l}}^{-} \omega\right|^{2}=0$ on $N$, that is, $d_{A_{l}}^{-} \omega=0$ at any point $x \in k(N)$. Therefore, $d_{A_{l}} \omega=0$ at $x \in k(N)$. In the case $m$ is odd, we have

$$
\left(\left|d_{A_{l}}^{+} \omega\right|^{2}-\left|d_{A_{l}}^{-} \omega\right|^{2}\right) \operatorname{vol}\left(g_{l}\right)=-i d_{A_{l}} \omega \wedge \overline{d_{A_{l}} \omega}=-i d_{A} \omega \wedge \overline{d_{A} \omega}=-i d\left(\omega \wedge d_{A} \bar{\omega}\right) .
$$

Thus $d_{A_{l}} \omega=0$ at $x \in k(N)$ follows from the same argument as above.

(2) If $d_{A_{l}} \omega=0$ at a point $x$, then it is 0 along the leaf of $H$ through $x$. This is because $0=d_{A}^{2} \omega=d_{A} d_{A_{l}} \omega=\tau \wedge \nabla_{A_{X}} d_{A_{l}} \omega+d_{A_{l}} d_{A_{l}} \omega$ on $U$, since $d_{A_{h}} \omega=0$ on $M$ and $A$ is flat. Here $U$ is an open neighbourhood of $x, X$ is a smooth section of $H$ and $\tau$ is a smooth section of $H^{*}$ such that $\langle X, \tau\rangle=1$ on $U$. Thus $\nabla_{A_{X}} d_{A_{l}} \omega=0$ on $U$, which implies the claim.

(3) By the above (1) and (2), we get $d_{A_{l}} \omega=0$ in an open subset of $M$. Now we claim $d_{A_{l}} \omega=0$ on the whole $M$. In the case $H$ is orientable, there is a nowhere zero section $\tau$ of $H^{*}$ over $M$. We can take a metric $g_{h}$ on $H^{*}$ such that $|\tau|=$ 1 , and take any metric $g_{l}$ on $L^{*}$ representing the $c_{l}$. Let $g=g_{h} \oplus g_{l}$ on $M$. Note that $d_{A_{l}} \omega=d_{A_{l}}^{-} \omega$ on $M$. Since $d_{A}\left(d_{A_{l}}^{-} \omega\right)=d_{A} d_{A} \omega=0$ and $d_{A}\left(* d_{A_{l}}^{-} \omega\right)=$ $d_{A}\left(\tau \wedge *_{l} d_{A_{l}}^{-} \omega\right)=d \tau \wedge *_{l} d_{A_{l}}^{-} \omega-\tau \wedge d_{A}\left(*_{l} d_{A_{l}}^{-} \omega\right)=\epsilon d \tau \wedge d_{A_{l}}^{-} \omega$, where $\epsilon$ is one of \pm 1 and $\pm i$, we can apply the unique extension theorem in [2] to get $d_{A_{l}} \omega=0$ on the whole $M$. In the case $H^{*}$ is non-orientable, the nowhere zero section $\tau$ of $H^{*}$ exists locally and we can take an open cover of $M$ and apply the above argument piece by piece.

Corollary 7.4. Let $\left(M, H, o_{l}, g_{l}, L, N\right)$ be as in Theorem 7.3. In the case $M$ is a closed smooth $(2 m+1)$-manifold, we have $H_{+}^{m-1}(M) \stackrel{\text { def }}{=} \frac{\text { Kerd }^{++}}{I m d} \cong \mathcal{H}^{m-1}(M)$. If in addition $M$ is oriented Riemannian, then we have $\mathcal{H}_{+}^{m-1}(M) \stackrel{\text { def }}{=} \operatorname{Kerd}^{++} \cap$ $\operatorname{Kerd}^{*} \cong \mathcal{H}^{m-1}(M) \cong H^{m-1}(M)$.

Theorem 7.3 and ([13], Corollary 5.7) together yield

Corollary 7.5. Let $M$ be a compact oriented smooth $(2 m+1)$-manifold with boundary and with the data $\left(H, o_{l}, o, g\right)$ as in Convention 1.1 such that $H \perp_{g} \partial M$. Then $\mathcal{H}_{+}^{m-1}(M, K)=\mathcal{H}^{m-1}(M, K) \cong H^{m-1}(M, K)$ and $b_{+}^{m-1}(M, K) \cong b^{m-1}(M, K)$ for any $K$.

Now we want to study $\operatorname{Ker}\left(d_{A}^{+s}\right)^{*}$ in $\Omega_{+}^{m+1}(E)$. Note that $\operatorname{Ker}\left(d_{A}^{+s}\right)^{*}=\operatorname{Ker}\left(d_{A}^{+}\right)^{*}$ for all nonzero $s$. Suppose $E$ is equipped with a $d_{A}$-parallel-invariant metric, so 
that we have (4.4). Since $H^{*}$ is oriented by the $o_{h}$, we can choose a nowhere zero section $\tau$ of $H^{*}$ over the whole $M$ such that $|\tau|=1$. Let $X$ be a section of $H$ such that $\langle X, \tau\rangle=1$ on $M$. For any $\omega \in \Omega_{+}^{m+1}(E)$ we have $\omega=\tau \wedge \omega^{+}$for a unique $\omega^{+} \in \Omega_{+}^{m}(E)$. By Lemma 4.3, we see that the equation $\left(d_{A}^{+s}\right)^{*} \omega=0$ on $M$, i.e., $\pi^{++} * d_{A} * \omega=0$ on $M$, is equivalent to the system of two equations:

$$
\begin{gathered}
d_{A_{l}} \omega^{+}=0 \text { on } M, \\
\pi_{l}^{+} *_{l} \nabla_{A_{X}} \omega^{+}=0 \text { on } M .
\end{gathered}
$$

Theorem 7.6. Let $M$ be a connected, smooth $(2 m+1)$-manifold with the data $\left(H, o_{l}, o, g\right)$ as in Convention 1.1, and let $A$ be any connection on a vector bundle $E$ with a $d_{A}$-parallel-invariant metric. Suppose $N$ is a smoothly imbedded $\mathbb{R}^{2 m}$ in $M$ which is transverse to $H$. Then $\left(d_{A}^{+s}\right)^{*} \omega=0$ on $M$ and $\left(\pi_{h} \omega\right)(x)=0$ at any point $x \in N$ if and only if $\omega=0$ on $M$.

Proof. Recall $\omega=\pi_{h} \omega$ for $\omega \in \Omega_{+}^{m+1}(E)$. Thus $\left(\pi_{h} \omega\right)(x)=0$ means $\left(i_{X} \omega\right)(x)=0$. There are two ways to propagate this vanishing on $N$ to the whole manifold $M$.

(1) Since $\left(i_{X} \omega\right)(x)=0$ at $x \in N, i_{X} \omega$ is 0 along the leaf of $H$ through $x$ in $M$. This is because (7.2) is a first order linear ordinary differential equation. For

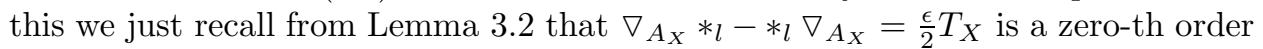
operator. Thus (7.2) has the leading term $\nabla_{A_{X}}\left(i_{X} \omega\right)$.

(2) Since $N$ is transverse to $\left.H\right|_{U}$, we get $i_{X} \omega=0$ on an open subset of $M$. This implies $i_{X} \omega=0$ on the whole $M$. Indeed, since $\left(d_{A}^{+s}\right)^{*} \omega=0$ on $M$, we have $\triangle_{m+1, A}^{+s} \omega \stackrel{\text { def }}{=} d_{A}^{+s}\left(d_{A}^{+s}\right)^{*} \omega=0$ on $M$. By using the unique extension theorem ([1], p.248, Remark 3), we get $i_{X} \omega=0$ on $M$, since $M$ is connected. (The principal symbol of $\triangle_{m+1, A}^{+s}$ at a covector $\xi$ is the multiplication of $-|\xi|^{2}$ when $|s|^{2}=2$. See Proposition 9.2.)

Here we emphasize that in Theorems 7.3 and 7.6 there are not any conditions on $T_{X}$ and $L$, and moreover $M$ can be either compact or noncompact.

Recall the $b$-boundary condition on $\omega \in \Omega_{+}^{m+1}(E)$ means $\omega_{h} \stackrel{\text { def }}{=} \pi_{h} \omega=0$ on $\partial M-K$. Thus we have

Corollary 7.7. Let $M$ be a compact oriented smooth $(2 m+1)$-manifold with boundary and with the data $\left(H, o_{l}, o, g\right)$ as in Convention 1.1, and let $A$ be a connection on a vector bundle $E$ with a $d_{A}$-parallel-invariant metric. Then for any $K \neq \partial M$, we have

$$
\mathcal{H}_{+}^{m+1}(A, K)=0, \quad b_{+}^{m+1}(A, K)=0 .
$$

Now we are going to get an estimate on $b_{+}^{m+1}(M, \partial M)$, where $M$ is a compact smooth $(2 m+1)$-manifold with boundary. This estimate has a geometric application in [7]. First we want to have a clear definition of the notions of $d_{A}$-holonomytranslation and $\left(d_{A}, S\right)$-holonomy-translation.

Let $M$ be a smooth oriented $(2 m+1)$-manifold with the data $\left(H, o_{l}, g_{l}\right)$, and $A$ a smooth connection on a vector bundle $E$ over $M$. In this section, for the sake of brevity, we let $\wedge^{+}$denote $\wedge_{+}^{m} L^{*} \otimes E$ over $M$, and let $\wedge^{j}$ denote one of $\wedge_{+}^{m} L^{*} \otimes E$ and $\wedge^{k} L^{*} \otimes E$ over $M$ for all $k$.

Let $J$ be a smoothly imbedded $[0,1]$ or $S^{1}$ in a leaf of $H$. Let $X$ be a section of $H$ over $J$. For a smooth section $\alpha$ of $\wedge^{j}$ over $J$, we can extend $\alpha$ to be a smooth section $\alpha^{\prime}$ of $\wedge^{j}$ over $M$, since $J$ is compact. Define $\nabla_{A_{X}} \alpha \stackrel{\text { def }}{=} \nabla_{A_{X}} \alpha^{\prime}$ at points of 
$J$. It is well-defined because $\nabla_{A_{X}}\left(f \alpha^{\prime}\right)=X(f) \alpha^{\prime}+f \nabla_{A_{X}} \alpha^{\prime}$, and $X(f)=0$ on $J$ if $f=1$ on $J$, where $f$ is a smooth function on $J$.

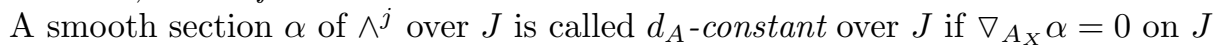
for all sections $X$ of $H$ over $J$. A section $\alpha$ of $\wedge^{+}$over $J$ is called $\left(d_{A}, S\right)$-constant over $J$ if $\pi_{l}^{+} *_{l} \nabla_{A_{X}} \alpha=0$ on $J$ for all sections $X$ of $H$ over $J$. Note the leading term of $\pi_{l}^{+} *_{l} \nabla_{A_{X}} \alpha=0$ is $\nabla_{A_{X}} \alpha$, so $\pi_{l}^{+} *_{l} \nabla_{A_{X}} \alpha=0$ is a first-order linear ordinary differential equation.

Let $\sigma:[0,1] \rightarrow J$ be a smooth path. For any $\alpha_{0} \in$ the fiber $\wedge_{\sigma(0)}^{j}$, there is a unique $d_{A^{-}}$-constant section $\alpha$ of $\wedge^{j}$ over $J$ such that $\alpha=\alpha_{0}$ at $\sigma(0)$. The map $T_{\sigma}: \wedge_{\sigma(0)}^{j} \rightarrow \wedge_{\sigma(1)}^{j}$ given by $T_{\sigma}\left(\alpha_{0}\right) \stackrel{\text { def }}{=} \alpha(\sigma(1))$ is called $d_{A}$-holonomy-translation along $\sigma$.

Similarly, for any $\alpha_{0} \in \wedge_{\sigma(0)}^{+}$, there is a unique $\left(d_{A}, S\right)$-constant section $\alpha^{\prime}$ of $\wedge^{+}$over $J$ such that $\alpha^{\prime}=\alpha_{0}$ at $\sigma(0)$. The map $T_{\sigma}^{S}: \wedge_{\sigma(0)}^{+} \rightarrow w_{\sigma(1)}^{+}$given by $T_{\sigma}^{S}\left(\alpha_{0}\right) \stackrel{\text { def }}{=} \alpha^{\prime}(\sigma(1))$ is called $\left(d_{A}, S\right)$-holonomy-translation along $\sigma$.

It is easy to show $T_{\sigma}$ and $T_{\sigma}^{S}$ depend only on the equivalent class of $\sigma$ modulo homotopies with the end points fixed. Thus they can be denoted as $T_{[\sigma]}$ and $T_{[\sigma]}^{S}$ respectively, where $[\sigma]$ denotes the homotopy class of $\sigma$.

Now we need to define another notion. Let $N$ be a smoothly imbedded codimension-one submanifold of $M$ which is transverse to $H$. Let $P(J)$ be the pseudogroup of paths $\sigma:[0,1] \rightarrow J$ modulo the equivalence of homotopies with the end points fixed. Let $\Gamma(H)$ be the union of $P(J)$ for all $J$ in the leaves of $H$. Define

$$
\Gamma(H, N)=\{[\sigma] \in \Sigma(H): \sigma(0) \in N \text { and } \sigma(1) \in N\} .
$$

It is called the holonomy pseudogroup $\Gamma(H, N)$ of $N$ induced by $H$.

According to Lemmas 7.1 and 7.2, $N$ has a unique smooth metric $g_{N}$ and an orientation $o_{N}$ to make $k^{*}:\left.L^{*}\right|_{N} \rightarrow T^{*} N$ as an orientation-preserving bundle isometry, where $k$ denotes the inclusion map from $N$ into $M$. In particular, $*_{N} k^{*} \alpha=k^{*} *_{l} \alpha$ for any $\left.\alpha \in \wedge^{j} L^{*}\right|_{N}$.

With the notations $T_{[\sigma]}, T_{[\sigma]}^{S}$ and $\Gamma(H, N)$ understood, we make the following definition.

Definition 7.8. A section $\alpha$ of $\left.\wedge_{+}^{m} T^{*} N \otimes E\right|_{N}$ over $N$ is called $\Gamma(H, N)$-invariant if $\left(k^{*}\right)^{-1} \alpha$ is preserved by $T_{[\sigma]}^{S}$ for any $\sigma \in \Gamma(H, N)$. A section $\alpha$ of $\left.\wedge^{j} T^{*} N \otimes E\right|_{N}$ over $N$ is called $\Gamma(H, N)$-invariant if $\left(k^{*}\right)^{-1} \alpha$ is preserved by $T_{[\sigma]}$ for any $\sigma \in \Gamma(H, N)$.

Remark 7.9. Note that we use the $T_{[\sigma]}^{S}$, not the $T_{[\sigma]}$, to define the notion of a $\Gamma(H, N)$-invariant section of $\left.\wedge_{+}^{m} T^{*} N \otimes E\right|_{N}$ over $N$. Of course, if $T_{X}=0$ for all $X \in H$, then $T_{[\sigma]}^{S}$ is the same as $T_{[\sigma]}$.

Recall that a connection $A$ on a vector bundle $E$ over $M$ restricts to a connection $\left.A\right|_{N}$ on $\left.E\right|_{N}$. Let $\Omega^{j}\left(N,\left.E\right|_{N}\right)$ denote the space of $\left.E\right|_{N}$-valued $j$-forms on $N$, and let $\Omega_{+}^{m}\left(N,\left.E\right|_{N}\right)$ denote the space of $\left.E\right|_{N}$-valued $m$-forms on $N$ such that $*_{N} \omega=\epsilon \omega$, where $\epsilon=1$ for even $m, \epsilon=i$ for odd $m$. Define $d_{\left.A\right|_{N}}^{+} \stackrel{\text { def }}{=} \pi_{N}^{+} d_{\left.A\right|_{N}}$, where $\pi_{N}^{+}=\frac{1+*_{N}}{2}$ is the projection from $\Omega^{m}\left(N,\left.E\right|_{N}\right)$ to $\Omega_{+}^{m}\left(N,\left.E\right|_{N}\right)$. Define 
2970

$$
\begin{aligned}
& \mathcal{H}_{+}^{m}\left(\left.A\right|_{N}\right) \stackrel{\text { def }}{=} \operatorname{Kerd}_{\left.A\right|_{N}}^{*_{N}} \text { in } \Omega_{+}^{m}\left(N,\left.E\right|_{N}\right), \\
& \mathcal{H}_{+}^{m-1}\left(\left.A\right|_{N}\right) \stackrel{\text { def }}{=} \operatorname{Kerd}_{\left.A\right|_{N}}^{+} \cap \operatorname{Kerd}_{\left.A\right|_{N}}^{*_{N}} \text { in } \Omega^{m-1}\left(N,\left.E\right|_{N}\right), \\
& \mathcal{H}^{j}\left(\left.A\right|_{N}\right) \stackrel{\text { def }}{=} \operatorname{Kerd}_{\left.A\right|_{N}} \cap \operatorname{Kerd}_{\left.A\right|_{N}}^{*_{N}} \text { in } \Omega^{j}\left(N,\left.E\right|_{N}\right) \text { for all } j .
\end{aligned}
$$

Also define

$$
\begin{aligned}
& \mathcal{H}_{\Gamma+}^{p}\left(\left.A\right|_{N}\right) \stackrel{\text { def }}{=} \text { the subgroup of } \Gamma \text {-invariant elements in } \mathcal{H}_{+}^{p}\left(\left.A\right|_{N}\right), \\
& \mathcal{H}_{\Gamma}^{j}\left(\left.A\right|_{N}\right) \stackrel{\text { def }}{=} \text { the subgroup of } \Gamma \text {-invariant elements in } \mathcal{H}^{j}\left(\left.A\right|_{N}\right) .
\end{aligned}
$$

where $\Gamma=\Gamma(H, N), p=m$ or $m-1$, and $0 \leq j \leq 2 m$. In the case $d_{A}=d$ we replace the term $\left.A\right|_{N}$ by $N$ in the above notations.

Now we return to study $k e r\left(d_{A}^{+s}\right)^{*}$ again. Recall (7.1) and (7.2). Since $*_{N} k^{*} \omega^{+}=$ $k^{*} *_{l} \omega^{+}=\epsilon k^{*} \omega^{+}, k^{*} \omega^{+}$is a $\Gamma(H, N)$-invariant element in $\Omega_{+}^{m}\left(N,\left.E\right|_{N}\right)$. Here $\epsilon=1$ for even $m, \epsilon=i$ for odd $m$.

Case (1). Suppose $H \perp_{g} N$, that is, $L$ is tangent to $N$. Then $\left\langle k_{*} T N, H^{*}\right\rangle=0$. Thus for any $j$-form $\omega$ on $M$, we have $k^{*} \pi_{l} \omega=k^{*} \omega$ at points of $N$. This is because $\left\langle k^{*} \pi_{h} \omega, \wedge^{j} T N\right\rangle=\left\langle\pi_{h} \omega, k_{*} \wedge^{j} T N\right\rangle=0$. Therefore (7.2) yields $0=k^{*} d_{A_{l}} \omega^{+}=$ $k^{*} d_{A} \omega^{+}=d_{\left.A\right|_{N}} k^{*} \omega^{+}$. So $k^{*} \omega^{+} \in \mathcal{H}_{\Gamma+}^{m}\left(\left.A\right|_{N}\right)$.

Case (2). Suppose $T_{X}=0$ on sections of $\left.\wedge_{+}^{m} L^{*}\right|_{N}$ over $N$ for $\left.X \in H\right|_{N}$. Then $\left(\nabla_{A_{X}} *_{l} \omega^{+}\right)(x)-\left(*_{l} \nabla_{A_{X}} \omega^{+}\right)(x)=\left(T_{X} \omega^{+}\right)(x)=0$ for any $x \in N$. Thus (7.2) restricted to $N$ becomes

$$
\nabla_{A_{X}}\left(\omega^{+}\right)=0 \text { on } N .
$$

This together with (7.1) gives

$$
d_{A}\left(\omega^{+}\right)=0 \text { on } N
$$

Thus $0=k^{*}\left(d_{A} \omega^{+}\right)(x)=\left(k^{*} d_{A} \omega^{+}\right)(x)=\left(d_{\left.A\right|_{N}} k^{*} \omega^{+}\right)(x)$ for all $x \in N$. So we also get $k^{*} \omega^{+} \in \mathcal{H}_{\Gamma+}^{m}\left(\left.A\right|_{N}\right)$.

Hence either in the case (1) or in the case (2), we have a well-definded group homomorphism

$$
r_{N}: \mathcal{H}_{+}^{m+1}(A) \rightarrow \mathcal{H}_{\Gamma+}^{m}\left(\left.A\right|_{N}\right) \quad \text { by } \quad r_{N} \omega \stackrel{\text { def }}{=} k^{*}\left(i_{X} \omega\right) .
$$

The condition of the case (1) that $H \perp_{g} N$ at points in $N$ can always be achieved by means of the deformation of the metric $g$ on $M$. In fact, let $g_{N}$ be any smooth positive definite metric on $N$, and let $g_{h}$ be any smooth fiberwise metric on $H$ over $M$. Then the fiberwise metric $g_{N}^{\prime}=g_{h} \oplus g_{N}$ on $\left.T M\right|_{N}=\left.H\right|_{N} \oplus T N$ can be smoothly extended to a metric $g^{\prime}$ on $T M$, because $N$ is smoothly imbedded in $M$. With respect to this $g^{\prime}, H \perp_{g^{\prime}} N$ at points in $N$.

Finally we can state the following result for $\operatorname{Ker}\left(d_{A}^{+s}\right)^{*}$, which rather easily follows from Theorem 7.6

Corollary 7.10. Let $\left(M, H, o_{l}, o, A\right)$ be the same as in Theorem 7.6. Let $N$ be a smoothly imbedded codimension-one submanifold of $M$ which is transverse to $H$.

(1) Then, for any smooth metric $g$ on $M$ such that $H \perp_{g} N$ or $T_{X}=0$ on $\left.\wedge^{+} L^{*}\right|_{N}$ over $N$ for $\left.X \in H\right|_{N}$, we have a well-definded injective group homomorphism $r_{N}$ defined by (7.6), and

$$
\operatorname{dimKer}\left(d_{A}^{+s}\right)^{*} \leq \operatorname{dim} \mathcal{H}_{\Gamma+}^{m}\left(\left.A\right|_{N}\right) \leq \operatorname{dim} \mathcal{H}_{+}^{m}\left(\left.A\right|_{N}\right),
$$

where $\Gamma=\Gamma(H, N)$.

(2) There always exist smooth metrics $g$ on $M$ such that $H \perp_{g} N$. 
Remark 7.11. According to Definitions 5.1 and 4.4, when $M$ is a compact oriented smooth $(2 m+1)$-manifold with non-empty boundary $\partial M$, we can replace $\operatorname{Ker}\left(d_{A}^{+s}\right)^{*}$ above by $\mathcal{H}_{+}^{m+1}(A, \partial M)$, and when $M$ is a closed oriented smooth $(2 m+1)$-manifold, we can replace $\operatorname{Ker}\left(d_{A}^{+s}\right)^{*}$ above by $\mathcal{H}_{+}^{m+1}(A)$.

If $T_{X}=0$ on level self-dual $m$-forms on $M$, then (7.5) holds on $M$. But note that the equation (7.5) and the space $\Omega_{+}^{m}(E)$ are independent of the data $L$. Thus we have

Proposition 7.12. Let $\left(M, H, o_{l}, o, g, A\right)$ be the same as Theorem 7.6. If $T_{X}=0$ on level self-dual $m$-forms on $M$ for all $X \in H$, then $\operatorname{Ker}\left(d_{A}^{+s}\right)^{*}$ is independent of $L$ and $s$.

\section{More computations of $(m+1)$-TH AND $m$-TH SELF-DUALITY COHOMOLOGY GROUPS}

The motivation of this section is to compute the $(m+1)$-th and $m$-th self-duality cohomology group of $N \times S^{1}$ and $N \times[0,1]$, where $N$ is a closed smooth oriented $2 m$-manifold.

For a subset $N$ of $M$ with a line field, we define the $H$-flow-closure of $N$ to be the union of the leaves of $H$ which go through $N$. This notion involves only the data $H$.

Theorem 8.1. Let $\left(M, H, o_{l}, o, g, A\right)$ be the same as in Theorem 7.6, and let $N$ be a smoothly imbedded codimension-one submanifold of $M$ which is transverse to $H$, $\Gamma=\Gamma(H, N)$. Assume that (1) $T_{X}=0$ on level self-dual $m$-forms on $M$; (2) $L$ is integrable over $M$; (3) $F_{A_{h}}=0$ on $M$; and (4) the $H$-flow-closure of $N$ is $M$, and $N$ is tangent to $L$. Then the map $r_{N}$ defined by (7.6) is an isomorphism:

$$
\operatorname{Ker}\left(d_{A}^{+s}\right)^{*} \cong \mathcal{H}_{\Gamma+}^{m}\left(\left.A\right|_{N}\right) .
$$

For notational conventions for the above $\operatorname{Ker}\left(d_{A}^{+s}\right)^{*}$, recall Remark 7.11. For the proof of the above theorem, we need some formulas in the case when $L$ is integrable over $M$. According to the Frobenius theorem, $L$ is integrable means that any point has a neighborhood $U$ on which there is a nonvanishing section $\nu$ of $\left.H^{*}\right|_{U}$ such that $d \nu=0$. Here we recall that $H^{*}=L^{\perp}$. By using Lemma 3.3, one can easily prove

Lemma 8.2. Let $\nu$ be a non-vanishing section of $\left.H^{*}\right|_{U}$ over an open subset $U$ such that $d \nu=0$, and $Y$ be a section of $\left.H\right|_{U}$ such that $\langle Y, \nu\rangle=1$ on $U$. Let $\alpha$ be an $E$-valued level $j$-form on $U, \beta$ be an E-valued level $(j-1)$-form on $U$, and $\gamma$ be a level form on $U$. Then

(1) $d_{A}(\nu \wedge \beta+\alpha)=\nu \wedge\left(\nabla_{A_{Y}} \alpha-d_{A_{l}} \beta\right)+d_{A_{l}} \alpha$ on $U$.

(2) $d_{A_{l}}^{2} \alpha=F_{A_{l}} \wedge \alpha$ and $\left(\nabla_{A_{Y}} d_{A_{l}}-d_{A_{l}} \nabla_{A_{Y}}\right) \alpha=F_{A_{Y}} \wedge \alpha$ on $U$.

(3) $d_{l}^{2} \gamma=0$ and $\nabla_{Y} d_{l} \gamma=d_{l} \nabla_{Y} \gamma$ on $U$.

Proof of Theorem 8.1. We give the proof in the case $m$ is even. (The proof for odd $m$ is completely similar.) Since $r_{N}$ is injective according to Theorem 7.6, we need only to prove it is surjective. Since the $H$-flow-closure of $N$ is $M$, we can take any $\omega_{0}^{+} \in \mathcal{H}_{\Gamma+}^{m}\left(\left.A\right|_{N}\right)$ and use $d_{A}$-holonomy-translation to get a well-defined level $m$-form $\eta$ on the whole $M$ such that $\eta(x)=\omega_{0}^{+}(x)$ for $x \in N$ and $\nabla_{A_{X}} \eta=0$ on $M$ as well. Since $H$ is orientable, we can choose a nowhere zero section $X$ of $H$ over $M$. 
(1) We need to prove $\eta \in \Omega_{+}^{m}(E)$. Since $T_{X}=0$, we have $\nabla_{A_{X}} *_{l} \eta=*_{l} \nabla_{A_{X}} \eta=0$ on $M$. Hence $\nabla_{A_{X}}\left(*_{l} \eta-\eta\right)=0$ on $M$. But on $N$, we know that $*_{l} \eta-\eta=$ $*_{l} \omega_{0}^{+}-\omega_{0}^{+}=0$. Thus $*_{l} \eta-\eta=0$ on $M$, since the $H$-flow-closure of $N$ is $M$.

(2) Secondly we need to prove $d_{A} \eta=0$ on $M$. Since $L$ is integrable and $F_{A_{h}}=0$, we get $\nabla_{A_{X}} d_{A_{l}} \eta=d_{A_{l}} \nabla_{A_{X}} \eta=0$ on $M$, according to Lemma 8.2. Recall that $k$ denotes the inclusion map from $N$ into $M$. We have

$$
\begin{aligned}
\left\langle d_{A_{l}} \eta,\left.\wedge^{m+1} L\right|_{N}\right\rangle & =\left\langle d_{A} \eta,\left.\wedge^{m+1} L\right|_{N}\right\rangle=\left\langle k^{*} d_{A} \eta, \wedge^{m+1} T N\right\rangle \\
& =\left\langle d_{\left.A\right|_{N}} k^{*} \eta, \wedge^{m+1} T N\right\rangle=\left\langle d_{\left.A\right|_{N}} \omega_{0}^{+}, \wedge^{m+1} T N\right\rangle=0
\end{aligned}
$$

at points of $N$. Thus $d_{A_{l}} \eta=0$ at points of $N$. This yields $d_{A_{l}} \eta=0$, since the $H$-flow-closure of $N$ is $M$.

Therefore according to (7.5), $\tau \wedge \eta \in \mathcal{H}_{+}^{m+1}(A)$, where $\tau$ is the section of $H^{*}$ such that $\langle\tau, X\rangle=1$ on $M$.

Theorem 8.1 can be applied to the special case $M=N \times S^{1}$, where $N$ is a closed oriented smooth $2 m$-manifold. But before we have clear statements, we need to recall the definitions of the twisted half De Rham complexes on an oriented Riemannian $2 m$-manifold $N$.

In the case $m$ is even, we have $*_{N}^{2}=1$ on the middle-dimensional $\wedge^{m} T^{*} N$, where $*_{N}$ is the star operator on $N$. Let $F$ be a real or complex vector bundle over $N$, $\Omega^{j}(N, F)$ the space of all sections of $\wedge^{j} T^{*} N \otimes F$, and $\Omega_{ \pm}^{m}(N, F)$ the space of all sections of $\wedge_{ \pm}^{m} T^{*} N \otimes F$, where $\wedge_{ \pm}^{m} T^{*} N$ are the \pm 1 -eigenbundle of $*_{N}$ respectively.

In the case $m$ is odd, we have $*_{N}^{2}=-1$ on $\wedge^{m} T^{*} N$. Let $\Omega^{j}(N, F)$ denote the space of all sections of $\wedge^{m} T_{c}^{*} N \otimes_{\mathbb{C}} F$, and let $\Omega_{ \pm}^{m}(N, F)$ denote the space of all sections of $\wedge_{ \pm}^{m} T_{c}^{*} N \otimes_{\mathbb{C}} F$, where $T_{c}^{*} N \stackrel{\text { def }}{=} \mathbb{C} \otimes_{\mathbb{R}} T^{*} N$ and $\wedge_{ \pm}^{m} T_{c}^{*} N$ are the $\pm i$ eigenbundle of $*_{N}$ respectively.

The twisted half De Rham complex on $N$ is:

$$
0 \rightarrow \Omega^{0}(N, F) \stackrel{d_{B}}{\rightarrow} \cdots \stackrel{d_{B}}{\rightarrow} \Omega^{m-1}(N, F) \stackrel{d_{B}^{+}}{\rightarrow} \Omega_{+}^{m}(N, F) \rightarrow 0,
$$

where $B$ is a flat connection on $F$ if $m \geq 3 ; B$ is an anti-self-dual connection on $F$ if $m=2$, which means $*_{N} F_{B}=-F_{B}$; and $B$ is an arbitrary connection on $F$ if $m=1$.

Definition 8.3. When $N$ is closed, the cohomology groups of complex (8.1) are denoted by $H^{j}(B), H_{+}^{m-1}(B)$ and $H_{+}^{m}(B)$ for $0 \leq j \leq m-2$; the space of harmonic forms at the $j$-th stage is denoted by $\mathcal{H}^{j}(B), \mathcal{H}_{+}^{m-1}(B), \mathcal{H}_{+}^{m}(B)$ for $0 \leq j \leq m-2$ respectively, and

$$
b_{+}^{m}(B) \stackrel{\text { def }}{=} \operatorname{dim} \mathcal{H}_{+}^{m}(B), \quad b_{+}^{m-1}(B) \stackrel{\text { def }}{=} \operatorname{dim} \mathcal{H}_{+}^{m-1}(B), \quad b^{j}(B) \stackrel{\text { def }}{=} \operatorname{dim} \mathcal{H}^{j}(B) .
$$

Here dim means the complex dimension when $m$ is odd, and means the real dimension when $m$ is even. When $d_{B}=d$ on $N$, we replace the term $B$ by $N$ and omit the term $F$. When the orientation of $N$ changes to its opposite, we change the + signs to - signs, and "anti-self-dual" to "self-dual" in all the corresponding notations. 
In the case $m$ is odd, we have

$$
\begin{array}{ll}
H_{-}^{m}(B)=\overline{H_{+}^{m}(B)}, & b_{-}^{m}(B)=b_{+}^{m}(B), \\
H_{-}^{m}(N)=\overline{H_{+}^{m}(N)}, & b_{-}^{m}(N)=b_{+}^{m}(N)=\frac{b^{m}(N)}{2}, \\
H_{ \pm}^{m-1}(N)=H^{m-1}(N), & \operatorname{dim}_{ \pm}^{m-1}(N)=b^{m-1}(N)
\end{array}
$$

for a closed oriented $2 m$-manifold with odd $m$. For example, when $N$ is a closed oriented 2-manifold, $b_{+}^{m}(N)$ is the genus of $N$.

In the case $m$ is even, we have

$$
\operatorname{dim}_{\mathcal{H}_{ \pm}^{m}}(N)=b_{ \pm}^{m}(N), \quad H_{ \pm}^{m-1}(N)=H^{m-1}(N), \quad \operatorname{dim} \mathcal{H}_{ \pm}^{m-1}(N)=b^{m-1}(N)
$$

for closed oriented $2 m$-manifold with even $m$, where $b_{+}^{m}(N)\left(b_{-}^{m}(N)\right.$ resp.) are the real dimensions of maximal positive ( negative resp.) subspace of the intersection form on $H^{m}(N)$.

Remark 8.4. Let $N$ be a connected, closed oriented smooth $2 m$-manifold with orientation $o_{N}$. Let $M=N \times S^{1}$ or $N \times[0,1]$ with the $H$ being the obvious line fields of tangents of copies of the circle $S^{1}$ or $[0,1]$. Then $L^{*}$ is the pullback of $T^{*} N$ to $M$. Let $o_{l}$ on $L^{*}$ be the pullback of $o_{N}$. Identify $N \times\{1\}$ with $N$ by $(x, 1) \rightarrow x$ and $N \times\{0\}$ with $N$ by $(x, 0) \rightarrow x$. Clearly, $\Gamma=\Gamma(H, N)$ is the trivial group. So

$$
\mathcal{H}_{\Gamma+}^{m}(N)=\mathcal{H}_{+}^{m}(N), \quad \mathcal{H}_{\Gamma+}^{m-1}(N)=\mathcal{H}_{+}^{m-1}(N) \cong H^{m-1}(N) .
$$

Let $A_{1}$ be a smooth connection on a vector bundle $F$ over $N=N \times\{1\}$. Let $A$ be the pullback of $A_{1}$ to $E=\pi_{N}^{*} F$ over $M: d_{A}=d_{A_{1}}+\frac{\partial}{\partial t} d t$, where $t \in[0,1]$ or is the angle parameter for $S^{1}$. For such a pullback connection $A$ of $A_{1}$, we have

$$
\begin{aligned}
& \mathcal{H}_{\Gamma+}^{m}\left(\left.A\right|_{N}\right) \cong \mathcal{H}_{+}^{m}\left(A_{1}\right) \stackrel{\text { def }}{=} \operatorname{ker}\left(d_{A_{1}}\right)^{*} \text { in } \Omega_{+}^{m}(N, F), \\
& \mathcal{H}_{\Gamma+}^{m-1}\left(\left.A\right|_{N}\right) \cong \mathcal{H}_{+}^{m-1}\left(A_{1}\right) \stackrel{\text { def }}{=} \operatorname{kerd} d_{A_{1}}^{+} \cap \operatorname{ker}\left(d_{A_{1}}\right)^{*} \text { in } \Omega^{m-1}(N, F) .
\end{aligned}
$$

Let $M$ take the orientation $o_{M}=d t \wedge o_{N}$. Then $\partial M=N \times\{1\} \sqcup N \times\{0\}$ gets the outward orientation $o_{\text {out }}$ defined by $e_{\text {out }} \wedge o_{\text {out }}=o_{M}$, where $e_{\text {out }}$ is a covector field on $\partial M$ pointing out of $M$. We have $o_{\text {out }}=o_{N}=o_{l}$ on $N \times\{1\}$, and $o_{\text {out }}=-o_{N}=-o_{l}$ on $N \times\{0\}$.

Now we can state the following results for the cases $M=N \times S^{1}$ and $M=$ $N \times[0,1]$, which are derived from Theorem 8.1 and Corollary 7.7.

Corollary 8.5. Let $M=N \times S^{1}, A_{1}, A$ as in Remark 8.4. Then

$$
\begin{array}{ll}
\mathcal{H}_{+}^{m+1}(A) \cong \mathcal{H}_{+}^{m}\left(A_{1}\right), & b_{+}^{m+1}(A)=b_{+}^{m}\left(A_{1}\right), \\
\mathcal{H}_{+}^{m+1}(M) \cong \mathcal{H}_{+}^{m}(N), & b_{+}^{m+1}(M)=b_{+}^{m}(N) .
\end{array}
$$

Corollary 8.6. Let $M=N \times[0,1], A_{1}, A$ as in Remark 8.4.

(1) If $K \neq \partial M$ then

$$
b_{+}^{m+1}(A, K)=b_{+}^{m+1}(M, K)=0 .
$$

(2) If $K=\partial M$ then

$$
\begin{array}{lll}
\mathcal{H}_{+}^{m+1}(A, \partial M) \cong \mathcal{H}_{+}^{m}\left(A_{1}\right), & b_{+}^{m+1}(A, \partial M)=b_{+}^{m}\left(A_{1}\right) ; \\
\mathcal{H}_{+}^{m+1}(M, \partial M) \cong \mathcal{H}_{+}^{m}(N), & b_{+}^{m+1}(M, \partial M)=b_{+}^{m}(N) .
\end{array}
$$


Now we compute $m$-th self-duality cohomology groups in some special situations. Recall that if a nowhere zero 1-form $\nu$ on $M$ is closed, that is, $d \nu=0$, then its dual tangent codimension-one plane field $L \stackrel{\text { def }}{=}\langle\nu\rangle^{\perp}$ is integrable. In such a case, we say that $L$ is defined by $\nu$. Let $Y$ be the vector field which is dual to $\nu$ by a metric $g$ on $M$, and $H$ be the tangent line field generated by $Y$. For brevity in the statements of Theorem 8.7 and 8.8, we make the following convention: referring to (4.4), we use $\mathcal{H}^{m}, \mathcal{H}^{m-1}, \mathcal{H}^{j}$ to denote $\mathcal{H}_{+s}^{m}(A), \mathcal{H}_{+}^{m-1}(A)$ and $\mathcal{H}^{j}(A)$ for all $0 \leq j \leq m-2$ respectively; referring to $(7.3)$, we use $\mathcal{H}_{N}^{m}, \mathcal{H}_{N}^{m-1}, \mathcal{H}_{N}^{j}$ to denote $\mathcal{H}_{\Gamma+}^{m}\left(\left.A\right|_{N}\right), \mathcal{H}_{\Gamma+}^{m-1}\left(\left.A\right|_{N}\right)$ and $\mathcal{H}_{\Gamma}^{j}\left(\left.A\right|_{N}\right)$ for all $0 \leq j \leq m-2$ respectively.

Theorem 8.7. Let $M$ be a connected, closed smooth $(2 m+1)$-manifold with the data $\left(H, o_{l}, o, g\right)$ as in Convention 1.1. Assume that (1) $H^{*}$ has a nowhere zero section $\nu$ over $M$ such that $d \nu=0$; (2) $T_{Y}=0$ on level $(m-1), m,(m+1)$ forms in the case $j=m$; the $T_{Y}=0$ on level $j,(2 m+1-j)$-forms in the case $0 \leq j \leq m-1$; (3) $N$ is a smoothly imbedded codimension-one submanifold of $M$ which is tangent to $L$; and (4) $A$ is a connection such that $F_{A_{h}}=0$ on a real or complex vector bundle $E$ with a $d_{A}$-parallel-invariant metric. Then

(1) The restriction mapping from $\mathcal{H}^{j}$ to $\mathcal{H}_{N}^{j-1} \oplus \mathcal{H}_{N}^{j}$ which sends $\nu \wedge \beta+\alpha$ to $\left(\left.\beta\right|_{N},\left.\alpha\right|_{N}\right)$ is a well-defined injective group homomorphism, and

$$
\operatorname{dim} \mathcal{H}^{j} \leq \operatorname{dim} \mathcal{H}_{N}^{j-1}+\operatorname{dim} \mathcal{H}_{N}^{j} .
$$

(2) If the $H$-flow-closure of $N$ is $M$, then the above restriction mapping is an isomorphism, and

$$
\mathcal{H}^{j} \cong \mathcal{H}_{N}^{j-1} \oplus \mathcal{H}_{N}^{j}
$$

Theorem 8.8. Assume the same conditions as in Theorem 8.7 except we here assume that $M$ is a compact connected smooth $(2 m+1)$-manifold with non-empty boundary $\partial M$ and $L$ is tangent to $\partial M$.

(1) If $K$ is empty, then the restriction mapping from $\mathcal{H}_{b}^{j}$ to $\mathcal{H}_{N}^{j}$ which sends $\tau \wedge \beta+\alpha$ to $\left.\alpha\right|_{N}$ is a well-defined injective homomorphism, and

$$
\operatorname{dim} \mathcal{H}_{b}^{j} \leq \operatorname{dim} \mathcal{H}_{N}^{j} .
$$

If $K=\partial M$, then the restriction mapping from $\mathcal{H}_{b}^{j}$ to $\mathcal{H}_{N}^{j-1}$ which sends $\tau \wedge \beta+\alpha$ to $\left.\beta\right|_{N}$ is a well-defined injective homomorphism, and

$$
\operatorname{dim} \mathcal{H}_{b}^{j} \leq \operatorname{dim} \mathcal{H}_{N}^{j-1} .
$$

(2) If the $H$-flow-closure of $N$ is $M$, then the above restriction mappings are isomorphisms,

$$
\begin{aligned}
& \mathcal{H}_{b}^{j} \cong \mathcal{H}_{N}^{j} \quad \text { if } K \text { is empty, } \\
& \mathcal{H}_{b}^{j} \cong \mathcal{H}_{N}^{j-1} \quad \text { if } K=\partial M .
\end{aligned}
$$

(3) If $K$ is nonempty and $K \neq \partial M$, then $\mathcal{H}_{b}^{j}=0$.

Remark 8.9. From the proof of Theorems 8.7 and 8.8 , which we will give at the end of this section, we can see that the conclusions of Theorem 8.7 still hold if we regard the $\mathcal{H}^{j}, \mathcal{H}_{N}^{j}$ as $\mathcal{H}^{j}(A), \mathcal{H}_{\Gamma}^{j}\left(\left.A\right|_{N}\right)$ respectively for all $j$ and all $\operatorname{dim} M$; and the conclusions of Theorem 8.8 also hold if we regard the $\mathcal{H}_{b}^{j}, \mathcal{H}_{N}^{j}$ as $\mathcal{H}^{j}(A, K)$, $\mathcal{H}_{\Gamma}^{j}\left(\left.A\right|_{N}\right)$ respectively for all $j$ and all $\operatorname{dim} M$. 
Corollary 8.10. Let $M=N \times S^{1}, A_{1}, A$ as in Remark 8.4, $0 \leq j \leq m-2$. Then

$$
\begin{array}{ll}
\mathcal{H}_{+s}^{m}(A) \cong \mathcal{H}_{+}^{m-1}\left(A_{1}\right) \oplus \mathcal{H}_{+}^{m}\left(A_{1}\right), & \mathcal{H}_{+}^{m-1}(A) \cong \mathcal{H}^{m-2}\left(A_{1}\right) \oplus \mathcal{H}_{+}^{m-1}\left(A_{1}\right), \\
\mathcal{H}^{j}(A) \cong \mathcal{H}^{j-1}\left(A_{1}\right) \oplus \mathcal{H}^{j}\left(A_{1}\right), & b_{+}^{m}(A)=b^{m-1}\left(A_{1}\right)+b_{+}^{m}\left(A_{1}\right), \\
b_{+}^{m-1}(A)=b^{m-1}\left(A_{1}\right)+b^{m-2}\left(A_{1}\right), & b^{j}(A)=b^{j-1}\left(A_{1}\right)+b^{j}\left(A_{1}\right) ; \\
\mathcal{H}_{+s}^{m}(M) \cong \mathcal{H}^{m-1}(N) \oplus \mathcal{H}_{+}^{m}(N), & \mathcal{H}_{+}^{m-1}(M) \cong \mathcal{H}^{m-1}(M), \\
b_{+}^{m}(M)=b^{m-1}(N)+b_{+}^{m}(N), & b_{+}^{m-1}(M)=b^{m-1}(M) .
\end{array}
$$

By this corollary, we get $\mathcal{H}_{+}^{m-1}(M) \cong H^{m-1}(M)$, which agrees with Corollary 7.4. On the other hand, the proof of Theorem 8.7 is quite different from that of Theorem 7.3. Recall that $\mathcal{H}^{j}\left(N \times S^{1}\right) \cong \mathcal{H}^{j-1}(N) \oplus \mathcal{H}^{j}(N)$ and $b^{j}\left(N \times S^{1}\right)=$ $b^{j-1}(N)+b^{j-2}(N)$ for all $j$.

Corollary 8.11. Let $M=N \times[0,1], A_{1}, A$ as in Remark 8.4, $0 \leq j \leq m-2$.

(1) If $K$ is empty, then

$$
\begin{gathered}
\mathcal{H}_{+s}^{m}(A) \cong \mathcal{H}_{+}^{m}\left(A_{1}\right), \quad \mathcal{H}_{+}^{m-1}(A) \cong \mathcal{H}^{m-1}\left(A_{1}\right), \quad \mathcal{H}^{j}(A) \cong \mathcal{H}^{j}\left(A_{1}\right), \\
b_{+}^{m}(A)=b_{+}^{m}\left(A_{1}\right), \quad b_{+}^{m-1}(A)=b^{m-1}\left(A_{1}\right), \quad b^{j}(A)=b^{j}\left(A_{1}\right) ; \\
\mathcal{H}_{+s}^{m}(M) \cong \mathcal{H}_{+}^{m}(N), \quad \mathcal{H}_{+}^{m-1}(M) \cong \mathcal{H}^{m-1}(N), \\
b_{+}^{m}(M)=b_{+}^{m}(N), \quad b_{+}^{m-1}(M)=b^{m-1}(N) .
\end{gathered}
$$

(2) If $K=\partial M$, then

$$
\begin{gathered}
\mathcal{H}_{+s}^{m}(A, \partial M) \cong \mathcal{H}^{m-1}\left(A_{1}\right), \quad \mathcal{H}_{+}^{m-1}(A, \partial M) \cong \mathcal{H}^{m-2}\left(A_{1}\right), \\
\mathcal{H}^{j}(A, \partial M) \cong \mathcal{H}^{j-1}\left(A_{1}\right), \quad b_{+}^{m}(A, \partial M)=b^{m-1}\left(A_{1}\right), \\
b_{+}^{m-1}(A, \partial M)=b^{m-2}\left(A_{1}\right), \quad b^{j}(A, \partial M) \cong b^{j-1}\left(A_{1}\right) ; \\
\mathcal{H}_{+s}^{m}(M, \partial M) \cong \mathcal{H}^{m-1}(N), \quad \mathcal{H}_{+}^{m-1}(M, \partial M) \cong \mathcal{H}^{m-2}(N), \\
b_{+}^{m}(M, \partial M)=b^{m-1}(N), \quad b_{+}^{m-1}(M, \partial M)=b^{m-2}(N) .
\end{gathered}
$$

(3) If $K$ is nonempty and $K \neq \partial M$, then

$$
\begin{gathered}
\mathcal{H}_{+s}^{m}(A, K)=\mathcal{H}_{+}^{m-1}(A, K)=\mathcal{H}^{j}(A, K)=\mathcal{H}_{+s}^{m}(M, K)=\mathcal{H}_{+}^{m-1}(M, K)=0, \\
b_{+}^{m}(A, K)=b_{+}^{m-1}(A, K)=b^{j}(A, K)=b_{+}^{m}(M, K)=b_{+}^{m-1}(M, K)=0 .
\end{gathered}
$$

Note the result for $H_{+}^{m-1}(M, K)$ in this corollary agrees with that of Corollary 7.5. For $M=N \times[0,1]$, recall that for all $j$ we have $\mathcal{H}^{j}(M) \cong \mathcal{H}^{j}(N)$, $b^{j}(M)=b^{j}(N) ; \mathcal{H}^{j}(M, \partial M)=\mathcal{H}^{j-1}(N), b^{j}(M, \partial M)=b^{j-1}(N) ; \mathcal{H}^{j}(M, K)=0$ and $b^{j}(M, K)=0$ if $K$ is nonempty and $K \neq \partial M$, all of which agree with the assertions of Theorem 8.8.

To prove Theorems 8.7 and 8.8, we need some formulas for the Laplacian operators in (5.4). The formulas look symbolically complicated but are structurally simple. Define

$$
\begin{aligned}
& \triangle_{m, A_{l}}^{+s}=|s|^{2} d_{A_{l}}^{+} d_{A}^{*_{l}}, \\
& \triangle_{m-1, A_{l}}^{+s}=d_{A_{l}} d_{A_{l}}^{*_{l}}+|s|^{2} d_{A}^{*_{l}} d_{A_{l}}^{+}, \\
& \triangle_{j, A_{l}}=d_{A_{l}} d_{A_{l}}^{*_{l}}+d_{A_{l}}^{*_{l}} d_{A_{l}},
\end{aligned}
$$


where $0 \leq j \leq m-2$. When $d_{A}=d$, we omit the terms $A, A_{l}$ in the above notations.

The first step in our computations for the Laplacian operators is the following lemma, which follows from Lemmas 4.3 and 8.2.

Lemma 8.12. Using the terms $\nu$ and $Y$ of Lemma 8.2, we have

$$
\begin{gathered}
d_{A}^{++s}(\nu \wedge \beta+\alpha)=\nu \wedge\left(\nabla_{A_{Y}} \alpha-d_{A_{l}} \beta\right)+s d_{A_{l}}^{+} \alpha, \\
d_{A}^{+s}\left(\nu \wedge \alpha+\gamma^{+}\right)=\nu \wedge\left(\pi_{l}^{+} \nabla_{A_{Y}} \gamma^{+}-s d_{A_{l}}^{+} \alpha\right),
\end{gathered}
$$

where $\beta$ is an E-valued level $(m-2)$-form on $U, \alpha$ is an E-valued level $(m-1)$-form on $U$ and $\gamma^{+}$is an E-valued level self-dual $m$-form on $U$. Moreover,

$$
\begin{aligned}
d_{A} *(\nu \wedge \beta+\alpha)=\nu & \wedge\left[\nabla_{A_{Y}}\left(|\nu| *_{l} \beta\right)+(-1)^{p+1} d_{A_{l}} \frac{1}{|\nu|} *_{l} \alpha\right]+d_{A_{l}}|\nu| *_{l} \beta, \\
* d_{A} *(\nu \wedge \beta+\alpha)= & (-1)^{n-p+1} \nu \wedge \frac{1}{|\nu|} *_{l} d_{A_{l}}|\nu| *_{l} \beta \\
& +\left(|\nu| *_{l} \nabla_{A_{Y}}\left(|\nu| *_{l} \beta\right)+(-1)^{p+1}|\nu| *_{l} d_{A_{l}} \frac{1}{|\nu|} *_{l} \alpha\right),
\end{aligned}
$$

where $\alpha$ is an E-valued level $j$-form on $U$ and $\beta$ is an E-valued level $(j-1)$-form on $U$.

The second step is to apply the above formulas to get the following identities. Note that the first two formulas apply to the case of $n=2 m+1$, and the third one applies to the case of any $n$.

Proposition 8.13. Assume $|\nu|=\kappa \neq 0$ is a constant and $F_{A_{Y}}=0$ on $U$. Let $\alpha$ be a level $(m-1)$-form on $U, \beta$ be a level $(m-2)$-form $\beta$ on $U, \gamma^{+}$be a level self-dual $m$-form on $U, \eta$ be a level $(j-1)$-form on $U$, and $\zeta$ be a level $j$-form on $U$.

(1) If $T_{Y}=0$ on level $(m-1), m,(m+1)$-forms, then

$\triangle_{m, A}^{+s}\left(\nu \wedge \alpha+\gamma^{+}\right)=\nu \wedge\left[-\kappa^{2} \nabla_{A_{Y}}^{2} \alpha+\triangle_{m-1, A_{l}}^{+s} \alpha\right]+\left[-\kappa^{2} \nabla_{A_{Y}}^{2} \gamma^{+}+\triangle_{m, A_{l}}^{+s} \gamma^{+}\right]$

and $\Delta_{m, A}^{+s}\left(\nu \wedge \alpha+\gamma^{+}\right)=0$ if and only if $\triangle_{m, A}^{+s}(\nu \wedge \alpha)=0, \quad \triangle_{m, A}^{+s}\left(\gamma^{+}\right)=0$.

(2) If $T_{Y}=0$ on level $(m-1),(m+2)$-forms, then

$\triangle_{m-1, A}^{+s}(\nu \wedge \beta+\alpha)=\nu \wedge\left[-\kappa^{2} \nabla_{A_{Y}}^{2} \beta+\triangle_{m-2, A_{l}} \beta\right]+\left[-\kappa^{2} \nabla_{A_{Y}}^{2} \alpha+\triangle_{m-1, A_{l}}^{+s} \alpha\right]$

and $\triangle_{m-1, A}^{+s}(\nu \wedge \beta+\alpha)=0$ if and only if $\triangle_{m-1, A}^{+s}(\nu \wedge \beta)=0, \quad \triangle_{m-1, A}^{+s} \alpha=0$.

(3) For all $j$ and all $n$, if $T_{Y}=0$ on level $j,(n-j)$-forms, then

$$
\triangle_{j, A}(\nu \wedge \eta+\zeta)=\nu \wedge\left[-\kappa^{2} \nabla_{A_{Y}}^{2} \eta+\triangle_{j-1, A_{l}} \eta\right]+\left[-\kappa^{2} \nabla_{A_{Y}}^{2} \zeta+\triangle_{j, A_{l}} \zeta\right] ;
$$$$
\text { and } \triangle_{j, A}(\nu \wedge \eta+\zeta)=0 \text { if and only if } \triangle_{j, A}(\nu \wedge \eta)=0, \quad \triangle_{j, A} \zeta=0 \text {. }
$$ 
Proof. Assume $\kappa=1$. If $\kappa \neq 1$, replace $\nu$ by $\frac{\nu}{\kappa}$. We apply (8.8) to prove the first formula. The other formulas can be proved in the same way. We have

$$
\begin{aligned}
& l_{s} d_{A}^{++} * d_{A} * l_{\bar{s}}\left(\nu \wedge \alpha+\gamma^{+}\right) \\
& =\nu \wedge\left[\nabla_{A_{Y}} *_{l} \nabla_{A_{Y}} *_{l} \alpha+(-1)^{m+1} \bar{s} \nabla_{A_{Y}} *_{l} d_{A_{l}} *_{l} \gamma^{+}+(-1)^{m+1} d_{A_{l}} *_{l} d_{A_{l}} *_{l} \alpha\right] \\
& \quad+s d_{A_{l}}^{+} *_{l} \nabla_{A_{Y}} *_{l} \alpha+(-1)^{m+1}|s|^{2} d_{A_{l}}^{+} *_{l} d_{A_{l}} *_{l} \gamma^{+}, \\
& h_{\bar{s}} \pi^{++} * d_{A} * d_{A}^{+} h_{s}\left(\nu \wedge \alpha+\gamma^{+}\right) \\
& =(-1)^{m+1} \nu \wedge\left[\bar{s} *_{l} d_{A_{l}} *_{l} \nabla_{A_{Y}} \gamma^{+}-|s|^{2} *_{l} d_{A_{l}} *_{l} d_{A_{l}}^{+} \alpha\right] \\
& +\pi_{l}^{+} *_{l} \nabla_{A_{Y}} *_{l} \nabla_{A_{Y}} \gamma^{+}-s \pi_{l}^{+} *_{l} \nabla_{A_{Y}} *_{l} d_{A_{l}}^{+} \alpha, \\
& \begin{aligned}
\triangle_{m, A}^{+s}\left(\nu \wedge \alpha+\gamma^{+}\right)\left[(-1)^{m} l_{s} d_{A}^{++} * d_{A} * l_{s}+(-1)^{m+1} h_{s} \pi^{++} * d_{A} * d_{A}^{+} h_{s}\right]\left(\nu \wedge \alpha+\gamma^{+}\right) \\
!
\end{aligned} \\
& \quad \nu \wedge\left[(-1)^{m} \nabla_{A_{Y}}^{2} *_{l}^{2} \alpha-d_{A_{l}} *_{l} d_{A_{l}} *_{l} \alpha-|s|^{2} *_{l} d_{A_{l}} *_{l} d_{A_{l}}^{+} \alpha\right] \\
& \quad+\left[(-1)^{m+1} *_{l}^{2} \nabla_{A_{Y}}^{2} \gamma^{+}-|s|^{2} d_{A_{l}}^{+} *_{l} d_{A_{l}} *_{l} \gamma^{+}\right] \\
& =\nu \wedge\left[-\nabla_{A_{Y}}^{2} \alpha+\triangle_{m-1, A_{l}}^{+s} \alpha\right]+\nu \wedge\left[-\nabla_{A_{Y}}^{2} \gamma^{+}+\triangle_{m, A_{l}}^{+s} \gamma^{+}\right] .
\end{aligned}
$$

In the above $\stackrel{!}{=}$, the cancellation

$$
-\bar{s} \nabla_{A_{Y}} *_{l} d_{A_{l} *_{l}} \gamma^{+}+\bar{s} *_{l} d_{A_{l}} *_{l} \nabla_{A_{Y}} \gamma^{+}=0
$$

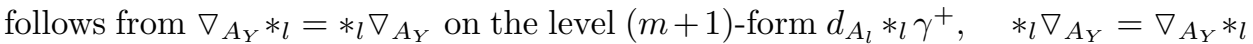
on the level $m$-form $\gamma^{+}$and $\nabla_{A_{Y}} d_{A_{l}}-d_{A_{l}} \nabla_{A_{Y}}=F_{A_{Y}}=0$ on the level $m$-form $\gamma^{+}$.

The cancellation

$$
\begin{aligned}
& (-1)^{m} s d_{A_{l}}^{+} *_{l} \nabla_{A_{Y}} *_{l} \alpha+(-1)^{m} s \pi_{l}^{+} *_{l} \nabla_{A_{Y}} *_{l} d_{A_{l}}^{+} \alpha \\
& \quad=(-1)^{m} s \pi_{l}^{+} d_{A_{l}} *_{l} \nabla_{A_{Y}} *_{l} \alpha+(-1)^{m} s \pi_{l}^{+} *_{l} \nabla_{A_{Y}} *_{l} \pi_{l}^{+} d_{A_{l}} \alpha=0
\end{aligned}
$$

follows from $\nabla_{A_{Y} *_{l}}=*_{l} \nabla_{A_{Y}}$ on the level $(m-1)$-form $\alpha, \quad *_{l}^{2}=(-1)^{m-1}$ on the level $(m-1)$-form $\nabla_{A_{Y}} \alpha, \quad \nabla_{A_{Y} *_{l}}=*_{l} \nabla_{A_{Y}}$ on the $m$-form $\pi_{l}^{+} d_{A_{l}} \alpha, \quad *_{l}^{2}=(-1)^{m}$ on the level $m$-form $\nabla_{A_{Y}} \pi_{l}^{+} d_{A_{l}} \alpha, \quad \nabla_{A_{Y}} \pi_{l}^{+}=\pi_{l}^{+} \nabla_{A_{Y}}$ on the level $m$-form $d_{A_{l}} \alpha$, and $\pi_{l}^{+}\left(d_{A_{l}} \nabla_{A_{Y}}-\nabla_{A_{Y}} d_{A_{l}}\right)=-\pi_{l}^{+} F_{A_{Y}}=0$ on the $(m-1)$-form $\alpha$.

Now we are ready to prove Theorems 8.7 and 8.8.

Proof of Theorem 8.7. We give the proof in the case $j=m$ and $m$ is even. The proof in other cases is similar.

Since $\mathcal{H}_{+}^{m}(A) \cong H_{+}^{m}(A)$ and $H_{+}^{m}(A)$ is independent of the metric $g_{h}$ on $H$, we can choose a metric $g_{h}^{\prime}$ on $H^{*}$ such that $|\nu|_{g_{h}^{\prime}}=1$, and work with the metric $g^{\prime}=g_{h}^{\prime} \oplus g_{l}$. By Proposition 8.13, $\triangle_{m, A}^{+s}\left(\nu \wedge \alpha+\gamma^{+}\right)=0$ if and only if $\triangle_{m, A}^{+s}(\nu \wedge \alpha)=0$ and $\triangle_{m, A}^{+s}\left(\gamma^{+}\right)=0$. Since $L_{Y} *_{l}-*_{l} L_{Y}=0$ on level $(m-1)$-forms and $m$-forms, we have $\nabla_{A_{Y}} *_{l} \alpha-*_{l} \nabla_{A_{Y}} \alpha=0$ and $\pi^{+} \nabla_{A_{Y}} \gamma^{+}=\nabla_{A_{Y}} \gamma^{+}$. Becasue $M$ is closed, we have the key fact in this proof:

$$
\triangle_{m, A}^{+s} \omega=0 \text { on } M \text { if and only if } d_{A}^{+} h_{s} \omega=0 \text { and } d_{A}^{*} l_{\bar{s}} \omega=0 \text { on } M .
$$

Thus we have

(1) $\triangle_{m, A}^{+s}(\nu \wedge \alpha)=0$ on $M$ if and only if $d_{A} * l_{\bar{s}}(\nu \wedge \alpha)=0$ and $d_{A}^{+} h_{s}(\nu \wedge \alpha)=0$ on $M$, which is equivalent to $\nabla_{A_{Y} *_{l}} \alpha=0, d_{A_{l} *_{l}} \alpha=0$ and $d_{A_{l}}^{+} \alpha=0$ on $M$; 
(2) $\triangle_{m, A}^{+s}\left(\gamma^{+}\right)=0$ on $M$ if and only if $d_{A}^{+} h_{s} \gamma^{+}=0$ and $d_{A} * l_{\bar{s}} \gamma^{+}=0$ on $M$, which is the same as $\pi^{+} \nabla_{A_{Y}} \gamma^{+}=0$ and $d_{A_{l}} *_{l} \gamma^{+}=0$ on $M$.

Now we can follow the same argument of the proof of Theorem 7.6 to get the statement (1) of Theorem 8.7, by using the above equivalent first order equations, and applying the unique extension theorem ([1], p. 248, Remark 3$)$ to the equations $\triangle_{m, A}^{+s}(\nu \wedge \alpha)=0$ and $\triangle_{m, A}^{+s} \gamma^{+}=0$.

Moreover, we can also follow the same argument of the proof of Theorem 8.1 to prove the statement (2) of Theorem 8.7, since the $H$-closure of $N$ is $M$.

Proof of Theorem 8.8. We give the proof in the case of $j=m$. The proofs in the other cases of Theorem 8.8 and Remark 8.9 are similar.

Let $\omega=\nu \wedge \alpha+\gamma^{+}$in $\mathcal{H}_{+}^{m}(A, K)$. Lemma 5.4 means that the second-order equation in Laplacians with the $\bar{b}$-boundary conditions is equivalent to the firstorder equations with the $b$-boundary conditions.

This equivalence corresponds to the statement (8.9) in the proof of Theorem 8.7, which allows us to use the same argument of that proof.

Thus we can claim: if $\alpha=0 \quad\left(\right.$ or $\left.\gamma^{+}=0\right)$ on a nonempty open subset of a leaf of the foliation of $L$, then $\alpha=0\left(\right.$ or $\left.\gamma^{+}=0\right)$ on the whole $M$. Since all components of $\partial M$ are leaves of the foliation of $L$, the definition of the $b$-boundary condition implies the statements (1) and (3) of Theorem 8.8.

Now we prove the statement (2) of Theorem 8.8. If $K$ is empty, then $\alpha=0$ on $\partial M$ and there is no boundary condition on $\gamma^{+}$. Thus the above claim implies $\alpha=0$ on the whole $M$. Since the $H$-flow-closure of $N$ is $M$, we can use the same argument of the proof of Theorem 8.1 to show that the restriction mapping is an ismorphism. A similar proof can be given in the case $K=\partial M$ : we just interchange $\gamma^{+}$and $\alpha$ in the above sentences.

The above argument has a by-product. Recall that, if the foliation of $H$ is Riemannian, then there is a smooth level-metric $g_{l}$ on the dual $L^{*} \stackrel{\text { def }}{=} H^{\perp}$ such that $T_{X}=0$ on the space of all the level forms. (See Proposition 6.1.) Also recall that a codimension-one foliation $\mathcal{L}$ admits some transversal Riemannian dimensionone foliation if and only if $\mathcal{L}$ is geodesible, namely, there is a Riemannian metric on $M$ such that all codimension-one leaves are totally-geodesic submanifolds ([11], p. 251). Hence by Remark 8.9, we have the following theorem.

Theorem 8.14. Let $M$ be a compact, connected, oriented smooth n-manifold with at least two boundary components. If there is a global nowhere zero closed 1form $\nu$ such that $\nu=0$ on $\partial M$ and the codimension-one foliation defined by $\nu$ is geodesible, then $H^{j}(M, K ; \mathbb{Q})=0$ for any nonempty union $K$ of components of $\partial M$ with $K \neq \partial M$, and any $j$.

In particular, if in addition $M$ is a connected, compact oriented smooth $n$ cobordism with two boundary components $N_{0}$ and $N_{1}$, then $\left(M ; N_{0}, N_{1}\right)$ is a rationalhomology-h-cobordism, that is, $H^{j}\left(M, N_{0} ; \mathbb{Q}\right)=0$ and $H^{j}\left(M, N_{1} ; \mathbb{Q}\right)=0$ in rational coefficient $\mathbb{Q}$ for all $j$.

\section{Proofs of Theorems 5.3, 5.8}

In this section, we prove Theorems 5.3 and 5.8. First, we need to do some basic computations about the symbols of the Laplacians on a half De Rham complex. In this section, we use the notations in the proof of Theorems 1.3, 1.6, 2.2 and 2.4 in Section 3. 
If $\rho \neq 0$ then every level $j$-covector $\alpha$ at a point has a unique decomposition

$$
\alpha=\rho \wedge \alpha_{\rho}+\alpha_{\bar{\rho}}
$$

such that $\alpha_{\rho} \in \wedge^{j-1} \rho^{\perp}$ and $\alpha_{\bar{\rho}} \in \wedge^{j} \rho^{\perp}$, where $\rho^{\perp}$ denotes the orthogonal complement in $L^{*}$ of the subspace $\langle\rho\rangle$ generated by $\rho \neq 0$. When $\rho=0$, we define $\alpha_{\bar{\rho}}=\alpha$ and $\alpha_{\rho}=0 . \quad \alpha_{\rho}$ and $\alpha_{\bar{\rho}}$ are uniquely determined by $\alpha$ and $\rho$.

Recall that $\alpha^{+} \stackrel{\text { def }}{=} \pi_{l}^{+} \alpha$ for $\alpha \in \wedge^{m} L^{*}$. Using the notations of the proof of Lemma 3.6, one can easily prove

Proposition 9.1. (1) $\sigma^{*}: \wedge^{j+1} T^{*} M \rightarrow \wedge^{j} T^{*} M$ is given by

$$
\sigma^{*}(\tau \wedge \beta+\alpha)=-\tau \wedge|\rho|^{2} \beta_{\rho}+k|\tau|^{2} \beta+|\rho|^{2} \alpha_{\rho}, \quad \text { where } \beta \in \wedge^{j} L^{*}, \alpha \in \wedge^{j+1} L^{*} .
$$

(2) If $\rho \neq 0$, then

$$
\begin{gathered}
\left((\rho \wedge \beta)^{+}\right)_{\rho}=\frac{1}{2} \beta_{\bar{\rho}} \quad \text { for } \beta \in \wedge^{j} L^{*}, \\
\left(\rho \wedge\left(\alpha^{+}\right)_{\rho}\right)_{\rho}=\frac{1}{2} \alpha^{+} \quad \text { for } \alpha^{+} \in \wedge_{+}^{m} L^{*} \text { or } \wedge_{+}^{m} L_{c}^{*} .
\end{gathered}
$$

It is well-known that the symbols of the Laplacian operators of the De Rham complex are the multiplication by $-|\xi|^{2}$. So $-\sigma_{\xi}\left(\triangle^{j}\right)(\omega)=|\xi|^{2} \omega$ for any $0 \leq j \leq$ $m-2, \omega \in \wedge^{j} T^{*} M$. For the other three Laplacians on a half De Rham complex, we have

Proposition 9.2. Let $\xi=\nu+\rho$ be a real nonzero covector at a point $x$ in $M$ with flow component $\nu$ and level component $\rho$. Let $\beta$ be a level $(m-2)$-covector, $\alpha$ be level $(m-1)$-covector, and $\gamma^{+}$be a level self-dual $m$-covector. Then

$$
\begin{gathered}
-\sigma_{\xi}\left(\triangle_{m-1}^{+s}\right)(\tau \wedge \beta+\alpha)=|\xi|^{2}\left(\tau \wedge \beta+\rho \wedge a_{\rho}\right)+\left(|\nu|^{2}+\frac{|s|^{2}}{2}|\rho|^{2}\right) a_{\bar{\rho}}, \\
-\sigma_{\xi}\left(\triangle_{m}^{+s}\right)\left(\tau \wedge \alpha+\gamma^{+}\right)=|\xi|^{2}\left(\tau \wedge \rho \wedge \alpha_{\rho}\right)+\left(|\nu|^{2}+\frac{|s|^{2}}{2}|\rho|^{2}\right)\left(\tau \wedge \alpha_{\bar{\rho}}+\gamma^{+}\right), \\
-\sigma_{\xi}\left(\triangle_{m+1}^{+s}\right)\left(\tau \wedge \gamma^{+}\right)=\left(|\nu|^{2}+\frac{|s|^{2}}{2}|\rho|^{2}\right)\left(\tau \wedge \gamma^{+}\right) .
\end{gathered}
$$

In particular,

$$
\text { when }|s|^{2}=2, \quad-\sigma_{\xi}\left(\triangle_{j}^{+s}\right)=|\xi|^{2},
$$

which is the symbol of the ordinary Laplacian operator. Here $j=m-1, m, m+1$.

Proof. We use the formula (3.3) to do the following computations. Let $\tau=k \tau$. If $\rho \neq 0$, then we can apply (2) of Proposition 9.1 to compute.

$$
\begin{aligned}
-\sigma_{\xi} & \left(\triangle_{m-1}^{+s}\right)(\tau \wedge \beta+\alpha)=\sigma \sigma^{*}(\tau \wedge \beta+\alpha)+\sigma^{*} l_{\bar{s}} l_{s} \sigma^{++}(\tau \wedge \beta+\alpha) \\
= & k \tau \wedge|\tau|^{2} k \beta+k \tau \wedge|\rho|^{2} \alpha_{\rho}-\rho \wedge \tau \wedge|\rho|^{2} \beta_{\rho}+\rho \wedge|\tau|^{2} k \beta+\rho \wedge|\rho|^{2} \alpha_{\rho} \\
& -\tau \wedge|\rho|^{2} k \alpha_{\rho}+\tau \wedge|\rho|^{2}(\rho \wedge \beta)_{\rho}+|\tau|^{2} k k \alpha-|\tau|^{2} k \rho \wedge \beta+|s|^{2}|\rho|^{2}\left((\rho \wedge \alpha)^{+}\right)_{\rho} \\
= & |\xi|^{2}(\tau \wedge \beta+\alpha)+\frac{|s|^{2}-2}{2}|\rho|^{2} a_{\bar{\rho}},
\end{aligned}
$$




$$
\begin{aligned}
& -\sigma_{\xi}\left(\triangle_{m}^{+s}\right)\left(\tau \wedge \alpha+\gamma^{+}\right)=l_{s} \sigma^{++} \sigma^{*} l_{\bar{s}}\left(\tau \wedge \alpha+\gamma^{+}\right)+h_{\bar{s}} \pi^{++} \sigma^{*} \sigma^{+} h_{s}\left(\tau \wedge \alpha+\gamma^{+}\right) \\
& =\tau \wedge k|\tau|^{2} k \alpha+\tau \wedge k|\rho|^{2} \bar{s}\left(\gamma^{+}\right)_{\rho}+\tau \wedge \rho \wedge|\rho|^{2} \alpha_{\rho}+s\left(\rho \wedge|\tau|^{2} k \alpha\right)^{+} \\
& \quad+s\left(\rho \wedge|\rho|^{2} \bar{s}\left(\gamma^{+}\right)_{\rho}\right)^{+}-\bar{s} \tau \wedge|\rho|^{2} k\left(\gamma^{+}\right)_{\rho} \\
& =|\xi|^{2}\left(\tau \wedge \alpha+\gamma^{+}\right)+\frac{|s|^{2}-2}{2}|\rho|^{2}\left(\tau \wedge \alpha_{\bar{\rho}}+\gamma^{+}\right) \\
& -\sigma_{\xi}\left(\triangle_{m+1}^{+s}\right)\left(\tau \wedge \gamma^{+}\right)=\sigma^{+} h_{s} h_{\bar{s}} \pi^{++} \sigma^{*}\left(\tau \gamma^{+}\right) \\
& =\tau \wedge k|\tau|^{2} k \gamma^{+}+\tau \wedge|s|^{2}\left[\rho \wedge|\rho|^{2}\left(\gamma^{+}\right)_{\rho}\right]^{+}=\left(|\tau|^{2}+\frac{|s|^{2}}{2}|\rho|^{2}\right)\left(\tau \wedge \gamma^{+}\right) .
\end{aligned}
$$

If $\rho=0$, then

$$
\begin{gathered}
-\sigma_{\xi}\left(\triangle_{m-1}^{+s}\right)(\tau \wedge \beta+\alpha)=\tau \wedge k k|\tau|^{2} \beta+\sigma^{*}(\tau \wedge k \alpha)=|\tau|^{2}(\tau \wedge \beta+\alpha), \\
-\sigma_{\xi}\left(\triangle_{m}^{+s}\right)\left(\tau \wedge \beta+\gamma^{+}\right)=l_{s}\left(\tau \wedge k k|\tau|^{2} \beta\right)+h_{\bar{s}}\left(k|\tau|^{2} k \gamma^{+}\right)=|\tau|^{2}\left(\tau \wedge \beta+\gamma^{+}\right), \\
-\sigma_{\xi}\left(\triangle_{m+1}^{+s}\right)\left(\tau \wedge \gamma^{+}\right)=\sigma^{+}\left(k|\tau|^{2} \gamma^{+}\right)=|\tau|^{2}\left(\tau \wedge \gamma^{+}\right) . \quad \square
\end{gathered}
$$

Proof of Theorem 5.3. Since ellipticity depends only on principal symbols, we need to prove Theorem 5.3 only for $d_{A}=d$.

(1) Let $x \in \partial M$ be any given point. Let $\tau_{x}$ be any fixed nonzero element in the fiber $H_{x}^{*}$ at $x$, and $\rho_{x}$ any fixed element in the fiber $L_{x}^{*}$ at $x$. Then ellipticity of a boundary system at $(x, \xi)$ with $\xi=t \tau_{x}+\rho_{x} \neq 0$ for all real numbers $t$ depends only on their principal symbols at $(x, \xi)$. In other words, if two boundary systems have the same principal symbols at $(x, \xi)$ with $\xi=t \tau_{x}+\rho_{x} \neq 0$ for all real $t$, and if one is elliptic at $(x, \xi)$ with $\xi=t \tau_{x}+\rho_{x} \neq 0$ for all real $t$, then so is the other one. This follows from the algebraic formulation in [3] of ellipticity of boundary systems.

(2) Let $\tau$ be a nonvanishing section of $H^{*}$ over an open neighbourhood $V$ of $x$ in $M$ with $\tau=\tau_{x}$ at $x$. Let $X$ be a section of $H$ over $V$ such that $\langle\tau, X\rangle=1$ on $V$. Taking a frame at $x$ for $L^{*}$ and using flows generated by $X$, we get a local orthonormal frame $\left(\rho^{1}, \ldots, \rho^{2 m}\right)$ for $L^{*}$ over $V$ such that $L_{X} \rho^{i}=0$. Any $\omega \in U^{j}$ over $V$ can be written as

$$
\omega=\tau \wedge \omega_{\tau}+\omega_{l}, \quad \omega_{\tau}=\sum_{|I|=j-1} \omega_{I} \rho^{I}, \quad \omega_{l}=\sum_{|J|=j} \omega_{J} \rho^{J},
$$

where $I$ and $J$ are ordered subsets of $\{1, \ldots, 2 m\},|J|$ is the length of $J$, and $\rho^{J}$ is the ordered wedge product of $\rho^{j}$ for $j \in J$. By using Lemmas 3.3, 4.3 and the fact that $L_{X} \rho^{i}=0$, we see that $\bar{b}$-boundary condition (5.3) has the same system of principal symbols as that of the following boundary condition:

$$
\omega_{J}=0 \text { and } L_{X}\left(|\tau| *_{l} \omega_{\tau}\right)=0 \text { on } K, \quad \omega_{I}=0 \text { and } L_{X} \omega_{J}=0 \text { on } \partial M-K .
$$

The principal symbol at $x$ of $L_{X}|\tau| *_{l}$ is the same as that of $|\tau| *_{l} L_{X}$. Thus we can replace $L_{X}\left(|\tau| *_{l} \omega_{\tau}\right)=0$ on $K$ by $L_{X} \omega_{I}=0$ on $K$. These are classical Dirichlet and Neumann boundary conditions. Proposition 9.2 tells us the principal symbols of $\triangle_{m-1}^{+s}, \triangle_{m}^{+s}$ and $\triangle_{m+1}^{+s}$ on $\omega_{I}$ and $\omega_{J}$ for the metric $g=g_{h} \oplus g_{l}$ are the same as, the principal symbols of the ordinary Laplacian on $\omega_{I}$ and $\omega_{J}$ for the metric $g$, or 
for the new metric $g_{s}$ near $x$. Here $|t \tau+\rho|_{g_{s}} \stackrel{\text { def }}{=}|t \tau|_{g}+\frac{|s|^{2}}{2}|\rho|_{g}$. Therefore by (1) above, we see Theorem 5.3 holds.

Theorem 5.3 implies that $\mathcal{H}_{b}^{j}$ is finite dimensional. Let $H_{j}$ be the projection from $U^{j}$ to $\mathcal{H}_{b}^{j}$. By standard theory for elliptic boundary systems, there is a fundamental solution $P_{j}(x, y, t)$, which is a symmetric double form in $x, y$. For each of $x, y$, it is an exterior form satisfying the $\bar{b}$-boundary condition and

$$
\frac{\partial P_{j}(x, y, t)}{\partial t}+\triangle_{j}^{x} P_{j}(x, y, t)=0
$$

for $x, y \in M, t>0$;

$$
\lim _{t \rightarrow 0+} \int_{M} P_{j}(x, y, t) \wedge * \omega(y)=\omega(x)
$$

for any $\omega \in U^{j}$; and in addition $P_{j}(x, y, t) \rightarrow 0$ uniformly for $x, y$ in $M$, as $t \rightarrow \infty$. The Green's operator $G_{j}: U^{j} \rightarrow U_{\bar{b}}^{j}$ is defined as

$$
\left(G_{j} \omega\right)(x)=\int_{0}^{\infty} d t \int_{M} P_{j}(x, y, t) \wedge *\left(\omega(y)-\left(H_{j} \omega\right)(y)\right) .
$$

Since $P_{j}(x, y, t)$ satisfies the $\bar{b}$-boundary conditions in $x$, the image of $G_{j}$ is contained in $U_{\bar{b}}^{j}$.

The following Lemma 9.3 can be proved by using the Green's formula (5.5) and Lemma 5.5.

Lemma 9.3. (1) $D_{j} G_{j} \omega=G_{j+1} D_{j} \omega$ is in $U_{\bar{b}}^{j}$ for any $\omega \in U^{j}$ such that $\omega_{l}=0$ on $K$.

(2) $D_{j-1}^{*} G_{j} \omega=G_{j-1} D_{j-1}^{*} \omega$ is in $U_{\bar{b}}^{j}$ for any $\omega \in U^{j}$ such that $\omega_{h}=0$ on $\partial M-K$.

Proof of Theorem 5.8. By using the Green's formula (5.5), we see that the right sides of (5.10) and (5.11) are direct sums. It is easy to prove that $\triangle_{j} G_{j} \omega=\omega-H_{j} \omega$ for any $\omega \in U^{j}$. Thus

$$
\omega=H_{j} \omega+\left(\omega-H_{j} \omega\right)=H_{j} \omega+D_{j-1} D_{j-1}^{*} G_{j}\left(\omega-H_{j} \omega\right)+D_{j}^{*} D_{j} G_{j}\left(\omega-H_{j} \omega\right) .
$$

Recall the image of $G_{j}$ is in $U_{\bar{b}}^{j}$. By using Lemma 5.5, we see the image of $D_{j-1}^{*} G_{j}$ is in $U_{b}^{j-1}$, and the image of $D_{j} G_{j}$ is in $U_{b}^{j+1}$. Therefore, (5.10) holds.

Lemma 5.5 proves the $\supset$ side of (5.11). If $\omega$ in $U_{b}^{j}$, then $D_{j-1}^{*} G_{j}\left(\omega-H_{j} \omega\right)$ $=G_{j-1} D_{j-1}^{*} \omega \in U_{\bar{b}}^{j-1}$ and $D_{j} G_{j}\left(\omega-H_{j} \omega\right)=G_{j+1} D_{j} \omega \in U_{\bar{b}}^{j+1}$ by Lemma 9.3. Thus (9.5) gives the $\subset$ side of (5.11).

Let $K e r$ denote $K \operatorname{Ker} D_{j}$ in $U_{\dot{b}}^{j}$, and let $I m$ denote $D_{j-1}\left(U_{\dot{b}}^{j-1}\right)$. Since $\operatorname{Ker} \subset U_{b}^{j}$ and $K e r \supset \mathcal{H}_{b}^{j} \oplus D_{j-1}\left(U_{\bar{b}}^{j-1}\right)$, and since $K e r$ is orthogonal to $D_{j}^{*}\left(U_{\bar{b}}^{j+1}\right)$, we get $K e r=\mathcal{H}_{b}^{j} \oplus D_{j-1}\left(U_{\bar{b}}^{j-1}\right)$. Because $D_{j-1}\left(U_{\bar{b}}^{j-1}\right) \subset I m, D_{j}^{*}\left(U_{\bar{b}}^{j+1}\right) \subset D_{j}^{*}\left(U_{\dot{b}}^{j+1}\right)$, and because $\operatorname{Im}$ and $D_{j}^{*}\left(U_{\dot{b}}^{j+1}\right)$ are mutually orthogonal with $\mathcal{H}_{b}^{j}$ in $U_{b}^{j}$, we get Im $=D_{j-1}\left(U_{\bar{b}}^{j-1}\right)$. Hence $(5.9)$ holds.

The proof is complete. 


\section{REFERENCES}

[1] N. Aronszajn, A unique continuation theorem for solutions of elliptic partial differential equations or inequalities of second order, J. de Mathématiques Pures et Appliquées 36 (1957), 235-249. MR 19:1056e

[2] N. Aronszajin, A. Krzywicki and J. Szarski, A unique continuation theorem for exterior differential forms on Riemannian manifolds, Arkiv för Matematik, Band 4 (1962), nr 34, 417-453. MR 25:3455

[3] M.F. Atiyah, The index theorem for manifolds with boundary, Appendix I in seminar on the Atiyah-Singer index theorem, Ann. Math. Stud. 57(1965), Princeton Univ. Press, 337-351. MR 33:6649

[4] M.F. Atiyah and I.M. Singer The index of elliptic operators: III, Ann. Math. 87(1968), 546-604. MR 38:5245

[5] L. Conlon, Transversally parallelizable foliations of codimension two, Trans. Amer. Math. Soc. 194 (1974), 79-102. MR 51:6844

[6] S.K. Donaldson and P.B. Kronheimer, The geometry of four-manifolds, Oxford Science Publications, Oxford, 1990. MR 92a:57036

[7] H.H. Fan, Self-dual vector fields on odd dimensional manifolds. Preprint, Yale, 1995.

[8] H.H. Fan, Gauge fields and line fields on five-manifolds. Preprint, Yale, 1995.

[9] H.H. Fan, Seiberg-Witten-type equation on five-manifolds. Preprint, Yale, 1995.

[10] J.F. Glazebrook, F.W. Kamber, H. Pedersen and A. Swann, Foliation reduction and selfduality, Geometric study of foliations, World Scientific Press, Singapore, 1994.

[11] P. Molino, Riemannian foliations, Birkhäuser, Boston, 1988. MR 89b:53054

[12] S. Nishikawa and H. Sato. On charateristic classes of Riemannian, conformal and projective foliations, J. Math. Soc. Japan 28 (1976), no. 2, 223-241. MR 53:4090

[13] D.B. Ray and I.M. Singer, R-torsion and the Laplacian on Riemannian manifolds, Advan. Math. 7 (1971), 145-210. MR 45:4447

[14] I. Vaisman, Conformal foliations, Kodai Math. J. 2 (1979), 26-37. MR 80g:57038

Department of Mathematics, Yale University, New Haven, Connecticut 06520

E-mail address: hhfan@math.yale.edu. 